\title{
Multi-scale asymptotic expansion for a small inclusion in elastic media
}

\author{
Makrem ARFAOUI, $^{*} \quad$ Mohamed Rafik BEN HASSINE, $^{\dagger} \quad$ Maher MOAKHER, ${ }^{\ddagger}$ \\ Yves RENARD, $\S \quad$ Gregory VIAL. $\$$
}

March 24, 2017

\begin{abstract}
The aim of this paper is to present an asymptotic expansion of the influence of a small inclusion of different stiffness in an elastic media. The applicative interest of this study is to provide tools to take into account this influence from the deformation without inclusion thanks to additive terms that can be precalculated and which depend only on the shape of the inclusion. We treat two problems: an anti-plane linearized elasticity problem and a plane strain one. On every expansion order we provide corrective terms modeling the influence of the inclusion using techniques of scaling and multi-scale asymptotic expansions. The built expansion is validated by comparing it to a test case obtained by solving the Poisson transmission problem in the case of a circular shape of the inclusion using variable separation method. Proofs of existence and uniqueness on unbounded domains of our fields are also adapted to the bidimensional Poisson problem and the linear elasticity one.
\end{abstract}

Keywords: Transmission problem, Asymptotic expansion, Multi-scale analysis, Infinite domains, linear elasticity, existence and unicity on unbounded domains.

\section{Introduction}

The interface problems investigated in this paper originate from a mechanical model for small inclusions coated with elastic bodies. Such arrangements are widely used for instance in tire's design in order to provide the desired structure stiffnesses. In this work, we consider a pair of two-dimensional approximations of the transmission problem between an elastic matrix and an

\footnotetext{
*Laboratoire de Mécanique Appliquée et Ingénierie, École Nationale d'Ingénieurs de Tunis, Université de Tunis El Manar, B.P. 37, 1002 Tunis-Belvédère, Tunisia. email: makrem.arfaoui@enit.utm.tn

${ }^{\dagger}$ INSA-Lyon, ICJ UMR5208, LaMCoS UMR5259, F-69621, Villeurbanne, France \& Laboratoire de Modélisation Mathématique et Numérique dans les Sciences de l'Ingénieur, École Nationale d'Ingénieurs de Tunis, Université de Tunis El Manar, B.P. 37, 1002 Tunis-Belvédère, Tunisia. email: mohamed-rafik.ben-hassine@insa-lyon.fr

${ }^{\ddagger}$ Laboratoire de Modélisation Mathématique et Numérique dans les Sciences de l’Ingénieur, École Nationale d'Ingénieurs de Tunis, Université de Tunis El Manar, B.P. 37, 1002 Tunis-Belvédère, Tunisia. email: maher.moakher@enit.rnu.tn

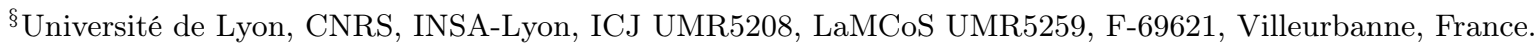
email: yves.renard@insa-lyon.fr

๑Université de Lyon, École Centrale de Lyon, CNRS UMR 5208, Institut Camille Jordan, 36 avenue Guy de Collongue, F-69134 Ecully Cedex, France. email: gregory.vial@ec-lyon.fr
} 
elastic small inclusion of different stiffnesses. The inclusions can be very small relatively to the characteristic size of the body and take them into account numerically with a good accuracy, for instance in a finite element calculation, can quickly become prohibitive for at least two reasons: a very fine mesh is required if the inclusion is small and the deformation induced by the inclusion being of non-regular nature, the approximation will be of poor quality. On the other side, homogenization techniques as in [1] can be very effective but restricted to a periodic or a quasi-perodic distribution of inclusions and thus to a relatively high density of inclusions. The objective is then to determine this influence by asymptotic analysis using techniques issued from $[2-7]$. The presented additive terms with respect to the deformation that does not account for inclusion can be used, for example, to be integrated in a numerical approximation after being pre-computed.

Some works have treated the case of perturbations whether for inclusions, for transmission problems essentially for conductivity [4] and Helmholtz equation $[5,6]$ or even for perforated domains and cavities. The case of perforated domains has been studied in [3,8-12] using techniques relying on the notion of profile, a normalized solution of the Laplace equation in the exterior domain obtained by blow-up of the perturbation. In fact, the influence of a boundary perforation of a smooth domain is studied in [3] and an asymptotic expansion of the shape function in terms of a size parameter is derived. The case of inclusions was treated for the first time by [13] giving a method to find the stresses set up in an elastic solid when the inclusion undergoes a change of form (for an ellipsoidal region whose elastic constants differ from those of the remaining material). The most important result is that the field inside the inclusion can be determined without having to find the field outside it. The resulting elastic field is found with the help of a sequence of imaginary cutting, straining and welding operators and using a biharmonic potential. The determination of the field outside the inclusion was briefly treated in this paper but studied deeply in [14] where the elastic field outside an ellipsoidal inclusion is expressed entirely in terms of the harmonic potential of a solid ellipsoid by replacing the biharmonic potential introduced in [13] by the harmonic potential of a certain surface distribution. Note that Eshelby's inclusion solution is one of the most significant contributions to micro-mechanics in the twentieth century and is based on the assumption that an inclusion is embedded in an unbounded ambient space. Schiavone alose tackled in [15] the case of an elliptic inhomogeneity embedded within an infinite isotropic elastic medium subjected to antiplane shear deformations under the assumption of non uniform loading matrix then extended the study with Antipov in [16] to the case of an inhomogeneity with the same assumptions but of an arbitrary shape and with an imperfect interface this time. A method is presented leading to the solution of the problem concerned with identifying the shape of the inhomogeneity and the form of the corresponding interface function which leads to an uniform stress field inside it. The analysis is based on complex variable methods. Li, Sauer and Wang deepened in [17] the precise characterization of the elastic fields due to inclusions in finite elastic media. It is important to say that, before the work done in [17], Kroner [18, 19] and Mazilu [20] tried to study the inclusion problem in a finite domain by searching a Green's function of Navier's equation in a finite domain. The attempt ended in failure because of the mathematical difficulty involved in obtaining a closed-form solution of Green's function in a finite domain.

On another side, Ammari and Kang described in [21] techniques for the reconstruction of small inclusions using only boundary measurements. These techniques rely on asymptotic expansions of the boundary perturbations due to the presence of the inclusions. The approach used to derive the asymptotic expansions is based on layer potential techniques allowing to deal with rough boundaries. By the mean of Muskhelishvili's complex variable method and the SchwartzChristoffel conformal mapping technique and motivated by the high stresses that occur at the 
junction between the fibers and the matrix in multi-fiber composite sheets which can cause failure, [22] presented a solution for determining the stresses in an infinite elastic plate containing a rigid rectangular inclusion subject to a uniform stress field. Using also the complex variable function method, the case of an arbitrary shape inclusion embedded in a half space is studied in [23]. Solutions are obtained by the mean of techniques of analytic extension, analytic continuation and conformal mapping. In [24] an asymptotic expansion of the difference between the displacement at the boundary with and without inclusion is presented (both the body and the inclusions are allowed to be anisotropic). To derive the asymptotic expansion, the authors follow the approach introduced by Capdeboscq and Vogelius in [25] (see also [26]) for the conductivity problem. Note that the formula obtained generalizes those already available in the case of homogeneous isotropic bodies with thin inhomogeneities of small diameters.

The outline of this paper is as follows: Two transmission problems of interest, a Poisson problem and a linear elasticity one, are formulated in Section 2. Asymptotic analyses for these two problems are presented in Sections 3 and 4, respectively. For each problem, existence, uniqueness and error estimates are established. Since we are led to deal with functions defined on infinite domains we reconsidered proof of existence and uniqueness using the generalized Hardy's inequalities and adequate weighted Sobolev spaces. Finally, some conclusions and perspectives are presented in Section 5.

\section{Formulation of the two transmission problems}

Before describing the approach we will develop, let us first present the two considered transmission problems: a scalar antiplane approximation and a linear plane strain approximation. Let us first define the domain of interest. Let $\Omega_{f}^{\varepsilon} \subset \mathbb{R}^{2}$ be a bounded connected domain of characteristic length $\varepsilon$ representing the inclusion (also called fiber) and being small enough compared to the characteristic size of the matrix represented by $\Omega_{m}^{\varepsilon} \subset \mathbb{R}^{2}$ of radius $R$ with smooth exterior boundary $\Gamma$. The asymptotic expansion being considered with respect to the parameter $\varepsilon$, we denote $\varepsilon \Omega_{f}^{1}$ the domain such that $\Omega_{f}^{\varepsilon}=\varepsilon \Omega_{f}^{1}$ and we will consider $\Omega_{f}^{\varepsilon}$ as a parametrized set of domains. The interface $\Gamma^{\varepsilon}=\partial \Omega_{f}^{\varepsilon}$ is also considered to be smooth. Fig. 1 makes explicit the geometrical setting.

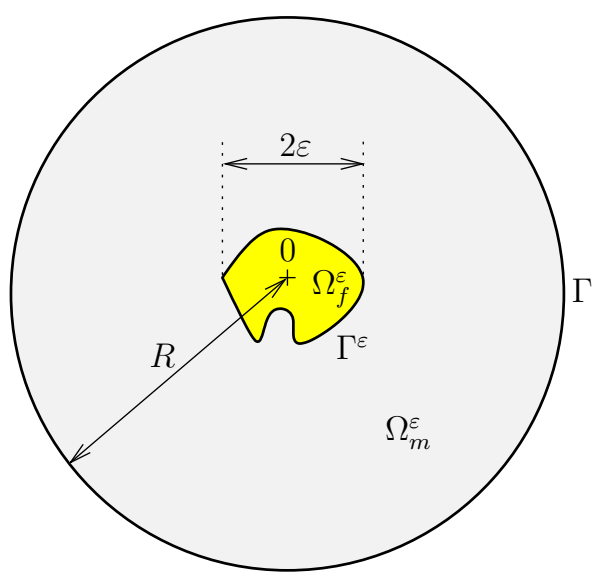

Figure 1: Initial domain $\Omega=\Omega_{f}^{\varepsilon} \cup \Gamma^{\varepsilon} \cup \Omega_{m}^{\varepsilon}$ with $\Omega_{f}^{\varepsilon}=\varepsilon \Omega_{f}^{1}$. Note that $\Gamma=\partial \Omega$ do not have necessarily to be circular. 
The antiplane problem reduces the mechanical problem to a Poisson one and is characterized by a displacement reduced to the normal component to the plane $\left(O, \boldsymbol{x}_{1}, \boldsymbol{x}_{2}\right)$ under the form $u_{3}=u\left(x_{1}, x_{2}\right)$. Transmission conditions consisting of the continuity conditions are set on the interface $\Gamma^{\varepsilon}$ and a non-homogeneous Dirichlet condition is set on the fixed boundary $\Gamma$. The strong formulation of the antiplane transmission problem reads:

$$
\begin{aligned}
\alpha_{f} \Delta u_{f}^{\varepsilon}=0 & \text { in } \Omega_{f}^{\varepsilon}, \\
\alpha_{m} \Delta u_{m}^{\varepsilon}=0 & \text { in } \Omega_{m}^{\varepsilon}, \\
u_{f}^{\varepsilon}=u_{m}^{\varepsilon} & \text { on } \Gamma^{\varepsilon}, \\
\alpha_{f} \partial_{\boldsymbol{n}} u_{f}^{\varepsilon}=\alpha_{m} \partial_{\boldsymbol{n}} u_{m}^{\varepsilon} & \text { on } \Gamma^{\varepsilon}, \\
u_{m}^{\varepsilon}=F & \text { on } \Gamma,
\end{aligned}
$$

where $\alpha_{f}$ and $\alpha_{m}$ are the shear moduli of the material in the inclusion and in the matrix, $u_{f}^{\varepsilon}$ is the solution of the problem in the inclusion area $\Omega_{f}^{\varepsilon}, u_{m}^{\varepsilon}$ is the solution of the problem in the matrix area $\Omega_{m}^{\varepsilon}, F$ is a prescribed displacement on $\Gamma$ and $\partial_{\boldsymbol{n}}$ denotes the normal derivative (outer for $\Omega_{f}^{\varepsilon}$, inner for $\Omega_{m}^{\varepsilon}$ ). For $F \in H^{\frac{1}{2}}(\Gamma)$, the existence and uniqueness of the solution of Problem (2.1) in a weak form are guaranteed by Lax-Milgram theorem.

The second transmission problem treated is a plane strain linear elasticity problem with two different fourth-order stiffness tensors $\boldsymbol{E}_{f}$ and $\boldsymbol{E}_{m}$ in the inclusion and in the matrix, respectively. The constitutive law relating the stress tensor $\boldsymbol{\sigma}_{k}$ to the strain tensor $\boldsymbol{\epsilon}_{k}$ is given by $\boldsymbol{\sigma}_{k}=\boldsymbol{E}_{k}: \boldsymbol{\epsilon}_{k}$, for $k \in\{f, m\}$. Transmission conditions consisting on the continuity conditions are still set on the interface $\Gamma^{\varepsilon}$ and a non-homogeneous Dirichlet one on the fixed boundary $\Gamma$. The strong formulation of the linear elasticity problem reads:

$$
\begin{aligned}
\operatorname{div} \boldsymbol{\sigma}_{f}\left(\boldsymbol{u}_{f}^{\varepsilon}\right)=\mathbf{0} & \text { in } \Omega_{f}^{\varepsilon}, \\
\operatorname{div} \boldsymbol{\sigma}_{m}\left(\boldsymbol{u}_{m}^{\varepsilon}\right)=\mathbf{0} & \text { in } \Omega_{m}^{\varepsilon}, \\
\boldsymbol{u}_{f}^{\varepsilon}=\boldsymbol{u}_{m}^{\varepsilon} & \text { on } \Gamma^{\varepsilon}, \\
\boldsymbol{\sigma}_{f}\left(\boldsymbol{u}_{f}^{\varepsilon}\right) \cdot \boldsymbol{n}_{f}=-\boldsymbol{\sigma}_{m}\left(\boldsymbol{u}_{m}^{\varepsilon}\right) \cdot \boldsymbol{n}_{m} & \text { on } \Gamma^{\varepsilon}, \\
\boldsymbol{u}_{m}^{\varepsilon}=\boldsymbol{F} & \text { on } \Gamma .
\end{aligned}
$$

where $\boldsymbol{u}_{f}^{\varepsilon}: \Omega_{f}^{\varepsilon} \longrightarrow \mathbb{R}^{2}$ is the solution of the problem in the inclusion domain $\Omega_{f}^{\varepsilon}, \boldsymbol{u}_{m}^{\varepsilon}: \Omega_{m}^{\varepsilon} \longrightarrow \mathbb{R}^{2}$ is the solution of the problem in the matrix domain $\Omega_{m}^{\varepsilon}$ and $\boldsymbol{F}$ is a prescribed displacement. The vectors $\boldsymbol{n}_{f}$ and $\boldsymbol{n}_{m}$ denote the outgoing unit normals to $\Omega_{f}^{\varepsilon}$ and $\Omega_{m}^{\varepsilon}$, respectively. The existence and uniqueness of the solution of a weak form of Problem (2.2) are also guaranteed by LaxMilgram theorem for a given $\boldsymbol{F}$ in $H^{\frac{1}{2}}\left(\Gamma ; \mathbb{R}^{2}\right)$.

\section{Asymptotic expansion for the antiplane problem}

The aim of this section is to build an asymptotic expansion $u_{\varepsilon, k}^{[K]}$ of a given order $K$ for the solution to the antiplane problem (2.1) having the following form for $k \in\{f, m\}$ :

$$
u_{\varepsilon, k}^{[K]}(\boldsymbol{x})=u^{(0)}(\boldsymbol{x})+\sum_{i=2}^{K} \varepsilon^{i} v^{(i)}(\boldsymbol{x})+\sum_{i=1}^{K} \varepsilon^{i} V_{k}^{(i-1)}\left(\frac{\boldsymbol{x}}{\varepsilon}\right),
$$

where $u^{(0)}$ is the solution to Problem (2.1) when $\varepsilon=0$ (i.e. without any inclusion), the functions $v^{(i)}$ are defined on $\Omega$ and the functions $V^{(i)}$ on the whole $\mathbb{R}^{2}$. 
Remark 3.1. An important aspect for the applications is that the functions $v^{(i)}$ and $V^{(i)}$, solutions to some problems which will be detailled further on, do not depend on $\varepsilon$ and depend linearly on $u^{(0)}$ only by the expansion of its gradient at $x=0$. For a given shape of the inclusion, this make it possible to pre-compute once for all a basis for each $v^{(i)}$ and $V^{(i)}$. This pre-computation can be done either analitically in the circular case (see Section 3.5.2), by an integral formulation using a Green kernel (as for instance in [27]) or by a numerical approximation with a boundary element method or a Galerkin method.

The rest of this section is subdivised into the following sub-sections: The asymptotic expansion construction is detailed in Section 3.1. Then, existence and uniqueness of the $V^{(i)}$ is stated in Section 3.2 and their behavior at infinity is specified in Section 3.3. This allows, in Section 3.4, to deduce an optimal error estimate for asymptotic expansion 3.1. Finally in Section 3.5 an explicit computation is performed using the separation of variable method in the case of a circular body and a circular inclusion. In addition to giving the explicit form of the expansion in this case, it allows us to compare the solution to its expansion and conclude that it is in accordance with the theoretical results.

\subsection{Building the asymptotic expansion}

We build the asymptotic expansion step by step. We need first to introduce the difference $d_{0, k}^{\varepsilon}=u_{k}^{\varepsilon}-u^{(0)}$ for $k \in\{f, m\}$ which is solution to:

$$
\begin{aligned}
\alpha_{f} \Delta d_{0, f}^{\varepsilon}(\boldsymbol{x})=0 & \text { in } \Omega_{f}^{\varepsilon}, \\
\alpha_{m} \Delta d_{0, m}^{\varepsilon}(\boldsymbol{x})=0 & \text { in } \Omega_{m}^{\varepsilon}, \\
d_{0, f}^{\varepsilon}(\boldsymbol{x})=d_{0, m}^{\varepsilon}(\boldsymbol{x}) & \text { on } \Gamma^{\varepsilon}, \\
\alpha_{f} \partial_{\boldsymbol{n}} d_{0, f}^{\varepsilon}(\boldsymbol{x})=\alpha_{m} \partial_{\boldsymbol{n}} d_{0, m}^{\varepsilon}(\boldsymbol{x})+\left(\alpha_{m}-\alpha_{f}\right) \partial_{\boldsymbol{n}} u^{(0)}(\boldsymbol{x}) & \text { on } \Gamma^{\varepsilon}, \\
d_{0, m}^{\varepsilon}(\boldsymbol{x})=0 & \text { on } \Gamma .
\end{aligned}
$$

Note the emergence of the term $\left(\alpha_{m}-\alpha_{f}\right) \partial_{\boldsymbol{n}} u^{(0)}$ in the transmission equation $(3.2 \mathrm{~d})$.

The original problem is posed on domains depending on $\varepsilon$. In order to work with a fixed domain and a fixed interface, we introduce as in $[2,3]$ the scaling $\boldsymbol{y}=\frac{\boldsymbol{x}}{\varepsilon}$. Let $\Omega^{m, \varepsilon}=\frac{\Omega_{m}^{\varepsilon}}{\varepsilon}$. Then, for $k \in\{f, m\}, D_{0, k}^{\varepsilon}(\boldsymbol{y})=d_{0, k}^{\varepsilon}(\boldsymbol{x})$ is the solution to the following scaled problem:

$$
\begin{array}{rc}
\alpha_{f} \Delta D_{0, f}^{\varepsilon}(\boldsymbol{y})=0 & \text { in } \Omega_{f}^{1}, \\
\alpha_{m} \Delta D_{0, m}^{\varepsilon}(\boldsymbol{y})=0 & \text { in } \Omega^{m, \varepsilon}, \\
D_{0, f}^{\varepsilon}(\boldsymbol{y})=D_{0, m}^{\varepsilon}(\boldsymbol{y}) & \text { on } \Gamma^{1}, \\
\alpha_{f} \partial_{\boldsymbol{n}} D_{0, f}^{\varepsilon}(\boldsymbol{y})=\alpha_{m} \partial_{\boldsymbol{n}} D_{0, m}^{\varepsilon}(\boldsymbol{y})+\varepsilon\left(\alpha_{m}-\alpha_{f}\right) \partial_{\boldsymbol{n}} u^{(0)}(\varepsilon \boldsymbol{y}) & \text { on } \Gamma^{1}, \\
D_{0, m}^{\varepsilon}(\boldsymbol{y})=0 & \text { on } \frac{\Gamma}{\varepsilon} .
\end{array}
$$

Now, as $\varepsilon$ is considered to be small compared to the size of the domain and to eliminate completely the dependence on $\varepsilon$, we approximate Problem (3.3) by the following problem posed on the dilated domain $\Omega^{\infty}=\lim _{\varepsilon \rightarrow 0} \Omega^{m, \varepsilon}$ as shown in Fig. 2:

$$
\begin{aligned}
\alpha_{f} \Delta D_{0, f}^{\varepsilon}(\boldsymbol{y})=0 & \text { in } \Omega_{f}^{1}, \\
\alpha_{m} \Delta D_{0, m}^{\varepsilon}(\boldsymbol{y})=0 & \text { in } \Omega^{\infty}, \\
D_{0, f}^{\varepsilon}(\boldsymbol{y})=D_{0, m}^{\varepsilon}(\boldsymbol{y}) & \text { on } \Gamma^{1}, \\
\alpha_{f} \partial_{\boldsymbol{n}} D_{0, f}^{\varepsilon}(\boldsymbol{y})=\alpha_{m} \partial_{\boldsymbol{n}} D_{0, m}^{\varepsilon}(\boldsymbol{y})+\varepsilon\left(\alpha_{m}-\alpha_{f}\right) \partial_{\boldsymbol{n}} u^{(0)}(\varepsilon \boldsymbol{y}) & \text { on } \Gamma^{1}, \\
D_{0, m}^{\varepsilon}(\boldsymbol{y}) \rightarrow 0 & \text { when }|\boldsymbol{y}| \rightarrow \infty .
\end{aligned}
$$




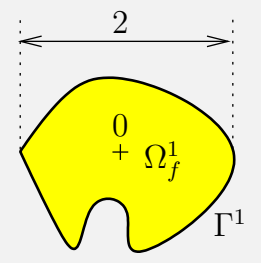

$\Omega^{\infty}$

Figure 2: Dilated geometry.

This allows us now to introduce the problem on the dilated geometry whose solutions will be the functions $V^{(p)}$ of the expansion (3.1):

$$
\begin{aligned}
\alpha_{f} \Delta V_{f}^{(p)}(\boldsymbol{y})=0 & \text { in } \Omega_{f}^{1}, \\
\alpha_{m} \Delta V_{m}^{(p)}(\boldsymbol{y})=0 & \text { in } \Omega^{\infty}, \\
V_{f}^{(p)}(\boldsymbol{y})=V_{m}^{(p)}(\boldsymbol{y}) & \text { on } \Gamma^{1}, \\
\alpha_{f} \partial_{\boldsymbol{n}} V_{f}^{(p)}(\boldsymbol{y})=\alpha_{m} \partial_{\boldsymbol{n}} V_{m}^{(p)}(\boldsymbol{y})+\varphi^{(p)}(\boldsymbol{y}) & \text { on } \Gamma^{1}, \\
V_{m}^{(p)}(\boldsymbol{y}) \rightarrow 0 & \text { when }|\boldsymbol{y}| \rightarrow \infty .
\end{aligned}
$$

The functions $V^{(p)}$ are introduced to offset terms appearing on the boundary of $\Gamma^{\varepsilon}$ where the data $\varphi^{(p)}(\boldsymbol{y})$ will be adapted for each order $p$. In fact, the smaller these terms, the smaller the residual and the expansion is driven to a higher order. The existence and uniqueness of the functions $V^{(p)}$ will be established later in Section 3.2. From the regularity assumptions on the domain, we can write a Taylor expansion of $\boldsymbol{\nabla} u^{(0)}(\varepsilon \boldsymbol{y})$ for $\boldsymbol{y} \in \Gamma^{1}$ :

$$
\boldsymbol{\nabla} u^{(0)}(\varepsilon \boldsymbol{y})=\sum_{i=0}^{K} \varepsilon^{i} \boldsymbol{\omega}_{0}^{(i)}(\boldsymbol{y})+O\left(\varepsilon^{K+1}\right)
$$

\subsubsection{First-order approximation}

The first-order approximation is constructed by compensating the first term in the expansion of $\left(\alpha_{m}-\alpha_{f}\right) \partial_{\boldsymbol{n}} u^{(0)}(\varepsilon \boldsymbol{y})$. With $V^{(0)}$ solving Problem (3.5) for $\varphi^{(0)}(\boldsymbol{y})=\left(\alpha_{m}-\alpha_{f}\right) \boldsymbol{\omega}_{0}^{(0)}(\boldsymbol{y}) \cdot \boldsymbol{n}$, where $\boldsymbol{\omega}_{0}^{(0)}(\boldsymbol{y})=\boldsymbol{\nabla} u^{(0)}(\mathbf{0})$, we update the residual:

$$
d_{1, k}^{\varepsilon}(\boldsymbol{x})=u_{k}^{\varepsilon}(\boldsymbol{x})-u^{(0)}(\boldsymbol{x})-\varepsilon V_{k}^{(0)}\left(\frac{\boldsymbol{x}}{\varepsilon}\right), \quad k \in\{f, m\} .
$$

The update of the difference allows us to obtain the first-order approximation:

$$
u_{\varepsilon, k}^{[1]}(\boldsymbol{x})=u^{(0)}(\boldsymbol{x})+\varepsilon V_{k}^{(0)}\left(\frac{\boldsymbol{x}}{\varepsilon}\right), \quad k \in\{f, m\} .
$$


Then, for $k \in\{f, m\}, d_{1, k}^{\varepsilon}$ is solution to:

$$
\begin{aligned}
\alpha_{f} \Delta d_{1, f}^{\varepsilon}(\boldsymbol{x})=0 & \text { in } \Omega_{f}^{\varepsilon}, \\
\alpha_{m} \Delta d_{1, m}^{\varepsilon}(\boldsymbol{x})=0 & \text { in } \Omega_{m}^{\varepsilon}, \\
d_{1, f}^{\varepsilon}(\boldsymbol{x})=d_{1, m}^{\varepsilon}(\boldsymbol{x}) & \text { on } \Gamma^{\varepsilon}, \\
\alpha_{f} \partial_{\boldsymbol{n}} d_{1, f}^{\varepsilon}(\boldsymbol{x})=\alpha_{m} \partial_{\boldsymbol{n}} d_{1, m}^{\varepsilon}(\boldsymbol{x})+\left(\alpha_{m}-\alpha_{f}\right)\left(\partial_{\boldsymbol{n}} u^{(0)}(\boldsymbol{x})-\omega_{0}^{(0)}(\boldsymbol{y})\right) & \text { on } \Gamma^{\varepsilon}, \\
d_{1, m}^{\varepsilon}(\boldsymbol{x})=-\varepsilon V_{m}^{(0)}\left(\frac{\boldsymbol{x}}{\varepsilon}\right) & \text { on } \Gamma .
\end{aligned}
$$

\subsubsection{Second-order approximation}

We have built a first-order approximation of the solution with $u^{(0)}$ and $V^{(0)}$ independent of $\varepsilon$. The next step is to build the second-order approximation. At this stage, we must do the same thing we did for the term that appeared on the interface $\Gamma^{\varepsilon}$ with the term that appears this time on $\Gamma$. In other words, we have two terms to compensate: $\left(\alpha_{m}-\alpha_{f}\right)\left(\partial_{\boldsymbol{n}} u^{(0)}-\omega_{0}^{(0)}\right)$ which has already been compensated in the first-order and that has yet to be, and the new term $-\varepsilon V_{m}^{(0)}\left(\frac{x}{\varepsilon}\right)$ on $\Gamma$. In fact, by analyzing things a little deeper, we can see that the term on $\Gamma^{\varepsilon}$ is of order $\varepsilon$ then it is in $O\left(\varepsilon^{2}\right)$ according to the $H^{-\frac{1}{2}}$ norm ( see (3.29)). The term on $\Gamma$ is of order $\varepsilon^{2}$ (expansion of $V$ at infinity see Section 3.3) and there is no scaling here so it is in $O\left(\varepsilon^{2}\right)$ according to the $H^{\frac{1}{2}}$ norm. By doing so we got the optimal error estimate in the previous first order approximation. So, to continue having this optimality the two terms have to be compensated in the same time from now on.

The functions introduced by Problem (3.5) are used to correct the term on $\Gamma^{\varepsilon}$ and we will introduce equivalent functions to play the same role but in this case on the boundary $\Gamma$. To this aim, we consider the following problem:

$$
\begin{array}{ll}
\alpha_{m} \Delta_{\boldsymbol{x}} v^{(q)}(\boldsymbol{x})=0 & \text { in } \Omega, \\
v^{(q)}(\boldsymbol{x})=\psi^{(q)}(\boldsymbol{x}) & \text { on } \Gamma .
\end{array}
$$

First, let us expand $V_{m}^{(0)}$ at infinity as follows:

$$
V_{m}^{(0)}(\boldsymbol{y})=\sum_{i=1}^{K} \frac{a_{0}^{(i)}(\theta)}{|\boldsymbol{y}|^{i}}+O\left(\frac{1}{|\boldsymbol{y}|^{K+1}}\right)=\sum_{i=1}^{K} \varepsilon^{i} \frac{a_{0}^{(i)}(\theta)}{|\boldsymbol{x}|^{i}}+O\left(\varepsilon^{K+1}\right), \quad \boldsymbol{x} \in \Gamma,
$$

where $\theta$ is the angular polar coordinate. This expansion at infinity is justified in Section 3.3. Now, with $V^{(1)}$ solution of Problem (3.5) for $\varphi^{(1)}(\boldsymbol{y})=\left(\alpha_{m}-\alpha_{f}\right) \boldsymbol{\omega}_{0}^{(1)}(\boldsymbol{y}) \cdot \boldsymbol{n}=\left(\alpha_{m}-\alpha_{f}\right) \omega_{0}^{(1)}(\boldsymbol{y})$ and $v^{(2)}$ solution of Problem (3.10) for $\psi^{(2)}(\boldsymbol{x})=-\frac{a_{0}^{(1)}(\theta)}{|\boldsymbol{x}|}$ we write the second-order term:

$$
d_{2, k}^{\varepsilon}(\boldsymbol{x})=d_{1, k}^{\varepsilon}(\boldsymbol{x})-\varepsilon^{2} v^{(2)}(\boldsymbol{x})-\varepsilon^{2} V_{k}^{(1)}\left(\frac{\boldsymbol{x}}{\varepsilon}\right), \quad k \in\{f, m\} .
$$

The update of the difference allows us to obtain the second-order approximation:

$$
u_{\varepsilon, k}^{[2]}(\boldsymbol{x})=u^{(0)}(\boldsymbol{x})+\varepsilon V_{k}^{(0)}\left(\frac{\boldsymbol{x}}{\varepsilon}\right)+\varepsilon^{2} v^{(2)}(\boldsymbol{x})+\varepsilon^{2} V_{k}^{(1)}\left(\frac{\boldsymbol{x}}{\varepsilon}\right), \quad k \in\{f, m\} .
$$


Then, for $k \in\{f, m\}, d_{2, k}^{\varepsilon}$ solves the following problem:

$$
\begin{array}{rr}
\alpha_{f} \Delta d_{2, f}^{\varepsilon}(\boldsymbol{x})=0 & \text { in } \Omega_{f}^{\varepsilon},(3.14 \mathrm{a}) \\
\alpha_{m} \Delta d_{2, m}^{\varepsilon}(\boldsymbol{x})=0 & \text { in } \Omega_{m}^{\varepsilon},(3.14 \mathrm{~b}) \\
d_{2, f}^{\varepsilon}(\boldsymbol{x})=d_{2, m}^{\varepsilon}(\boldsymbol{x}) & \text { on } \Gamma^{\varepsilon},(3.14 \mathrm{c}) \\
\alpha_{f} \partial_{\boldsymbol{n}} d_{2, f}^{\varepsilon}(\boldsymbol{x})=\alpha_{m} \partial_{\boldsymbol{n}} d_{2, m}^{\varepsilon}(\boldsymbol{x})+\left(\alpha_{m}-\alpha_{f}\right)\left(\partial_{\boldsymbol{n}} u^{(0)}(\varepsilon \boldsymbol{y})-\omega_{0}^{(0)}(\boldsymbol{y})-\varepsilon \omega_{0}^{(1)}(\boldsymbol{y})\right) & \\
+\varepsilon^{2}\left(\alpha_{m}-\alpha_{f}\right)\left(\partial_{\boldsymbol{n}} v^{(2)}(\varepsilon \boldsymbol{y})\right) & \text { on } \Gamma^{\varepsilon},(3.14 \mathrm{~d}) \\
d_{2, m}^{\varepsilon}(\boldsymbol{x})=\varepsilon\left(\frac{a_{0}^{(1)}(\theta)}{|\boldsymbol{x}|}-V_{m}^{(0)}\left(\frac{\boldsymbol{x}}{\varepsilon}\right)\right)-\varepsilon^{2} V_{m}^{(1)}\left(\frac{\boldsymbol{x}}{\varepsilon}\right) & \text { on } \Gamma . \quad(3.14 \mathrm{e})
\end{array}
$$

\subsubsection{Third-order approximation}

We expand as done before $\boldsymbol{\nabla} v^{(2)}(\varepsilon \boldsymbol{y})$ as follows:

$$
\boldsymbol{\nabla} v^{(2)}(\varepsilon \boldsymbol{y})=\sum_{i=0}^{K} \varepsilon^{i} \boldsymbol{\omega}_{2}^{(i)}(\boldsymbol{y})+O\left(\varepsilon^{K+1}\right)
$$

We expand too $V_{m}^{(1)}$ (see Section 3.3):

$$
V_{m}^{(1)}(\boldsymbol{y})=\sum_{i=1}^{K} \frac{a_{1}^{(i)}(\theta)}{|\boldsymbol{y}|^{i}}+O\left(\frac{1}{|\boldsymbol{y}|^{K+1}}\right)=\sum_{i=1}^{K} \varepsilon^{i} \frac{a_{1}^{(i)}(\theta)}{|\boldsymbol{x}|^{i}}+O\left(\varepsilon^{K+1}\right), \quad \boldsymbol{x} \in \Gamma .
$$

With $V^{(2)}$ solution of Problem (3.5) for $\varphi^{(2)}(\boldsymbol{y})=\left(\alpha_{m}-\alpha_{f}\right)\left(\boldsymbol{\omega}_{0}^{(2)}(\boldsymbol{y})+\boldsymbol{\omega}_{2}^{(0)}(\boldsymbol{y})\right) \cdot \boldsymbol{n}=\left(\alpha_{m}-\right.$ $\left.\alpha_{f}\right)\left(\omega_{0}^{(2)}(\boldsymbol{y})+\omega_{2}^{(0)}(\boldsymbol{y})\right)$ and $v^{(3)}$ solution of Problem (3.10) for $\psi^{(3)}(\boldsymbol{x})=-\frac{a_{1}^{(1)}(\theta)}{|\boldsymbol{x}|}-\frac{a_{0}^{(2)}(\theta)}{|\boldsymbol{x}|^{2}}$ we deduce the third-order residual:

$$
d_{3, k}^{\varepsilon}(\boldsymbol{x})=d_{2, k}^{\varepsilon}(\boldsymbol{x})-\varepsilon^{3} v^{(3)}(\boldsymbol{x})-\varepsilon^{3} V_{k}^{(2)}\left(\frac{\boldsymbol{x}}{\varepsilon}\right), \quad k \in\{f, m\} .
$$

The update of the difference allows us to obtain the third-order approximation:

$$
u_{\varepsilon, k}^{[3]}(\boldsymbol{x})=u^{(0)}(\boldsymbol{x})+\varepsilon V_{k}^{(0)}\left(\frac{\boldsymbol{x}}{\varepsilon}\right)+\varepsilon^{2} V_{k}^{(1)}\left(\frac{\boldsymbol{x}}{\varepsilon}\right)+\varepsilon^{2} v^{(2)}(\boldsymbol{x})+\varepsilon^{3} v^{(3)}(\boldsymbol{x})+\varepsilon^{3} V_{k}^{(2)}\left(\frac{\boldsymbol{x}}{\varepsilon}\right), \quad k \in\{f, m\} .
$$

Then, for $k \in\{f, m\}, d_{3, k}^{\varepsilon}$ solves the following problem:

$$
\begin{array}{rr}
\alpha_{f} \Delta d_{3, f}^{\varepsilon}(\boldsymbol{x})=0 & \text { in } \Omega_{f}^{\varepsilon,}, \\
\alpha_{m} \Delta d_{3, m}^{\varepsilon}(\boldsymbol{x})=0 & \text { in } \Omega_{m}^{\varepsilon}, \\
d_{3, f}^{\varepsilon}(\boldsymbol{x})=d_{3, m}^{\varepsilon}(\boldsymbol{x}) & \text { on } \Gamma^{\varepsilon}, \\
\alpha_{f} \partial_{\boldsymbol{n}} d_{3, f}^{\varepsilon}(\boldsymbol{x})=\alpha_{m} \partial_{\boldsymbol{n}} d_{3, m}^{\varepsilon}(\boldsymbol{x}) & \\
+\left(\alpha_{m}-\alpha_{f}\right)\left(\partial_{\boldsymbol{n}} u^{(0)}(\varepsilon \boldsymbol{y})-\omega_{0}^{(0)}(\boldsymbol{y})-\varepsilon \omega_{0}^{(1)}(\boldsymbol{y})-\varepsilon^{2} \omega_{0}^{(2)}(\boldsymbol{y})\right) & \\
+\varepsilon^{2}\left(\alpha_{m}-\alpha_{f}\right)\left(\partial_{\boldsymbol{n}} v^{(2)}(\varepsilon \boldsymbol{y})-\omega_{2}^{(0)}(\boldsymbol{y})\right) & \\
+\varepsilon^{3}\left(\alpha_{m}-\alpha_{f}\right)\left(\partial_{\boldsymbol{n}} v^{(3)}(\varepsilon \boldsymbol{y})\right) & \text { on } \Gamma^{\varepsilon}, \\
d_{3, m}^{\varepsilon}(\boldsymbol{x})=\varepsilon\left(\varepsilon^{2} \frac{a_{0}^{(2)}(\theta)}{|\boldsymbol{x}|^{2}}-\varepsilon \frac{a_{0}^{(1)}(\theta)}{|\boldsymbol{x}|}-V_{m}^{(0)}\left(\frac{\boldsymbol{x}}{\varepsilon}\right)\right) & \\
+\varepsilon^{2}\left(\varepsilon \frac{a_{1}^{(1)}(\theta)}{|\boldsymbol{x}|}-V_{m}^{(1)}\left(\frac{\boldsymbol{x}}{\varepsilon}\right)\right)-\varepsilon^{3} V_{m}^{(2)}\left(\frac{\boldsymbol{x}}{\varepsilon}\right) & \text { on } \Gamma .
\end{array}
$$




\subsubsection{Fourth-order approximation}

In order to conclude about the general form of functions $\varphi^{(n)}$ involved in problems solved by $V^{(n)}$ and functions $\psi^{(n)}$ involved in problems solved by $v^{(n)}$ we push the expansion to the fourth order. We expand as done before $\boldsymbol{\nabla} v^{(3)}(\varepsilon \boldsymbol{y})$ as follows:

$$
\boldsymbol{\nabla} v^{(3)}(\varepsilon \boldsymbol{y})=\sum_{i=0}^{K} \varepsilon^{i} \boldsymbol{\omega}_{3}^{(i)}(\boldsymbol{y})+O\left(\varepsilon^{K+1}\right)
$$

and $V_{m}^{(2)}$ as follows (see Section 3.3):

$$
V_{m}^{(2)}(\boldsymbol{y})=\sum_{i=1}^{K} \frac{a_{2}^{(i)}(\theta)}{|\boldsymbol{y}|^{i}}+O\left(\frac{1}{|\boldsymbol{y}|^{K+1}}\right)=\sum_{i=1}^{K} \varepsilon^{i} \frac{a_{2}^{(i)}(\theta)}{|\boldsymbol{x}|^{i}}+O\left(\varepsilon^{K+1}\right), \quad \boldsymbol{x} \in \Gamma .
$$

With $V^{(3)}$ solution of Problem (3.5) for $\varphi^{(3)}(\boldsymbol{y})=\left(\alpha_{m}-\alpha_{f}\right)\left(\boldsymbol{\omega}_{0}^{(3)}(\boldsymbol{y})+\boldsymbol{\omega}_{2}^{(1)}(\boldsymbol{y})+\boldsymbol{\omega}_{3}^{(0)}(\boldsymbol{y})\right)$. $\boldsymbol{n}=\left(\alpha_{m}-\alpha_{f}\right)\left(\omega_{0}^{(3)}(\boldsymbol{y})+\omega_{2}^{(1)}(\boldsymbol{y})+\omega_{3}^{(0)}(\boldsymbol{y})\right)$ and $v^{(4)}$ solution of Problem (3.10) for $\psi^{(4)}(\boldsymbol{x})=$ $-\frac{a_{2}^{(1)}(\theta)}{|\boldsymbol{x}|}-\frac{a_{1}^{(2)}(\theta)}{|\boldsymbol{x}|^{2}}-\frac{a_{0}^{(3)}(\theta)}{|\boldsymbol{x}|^{3}}$ we deduce the fourth-order residual:

$$
d_{4, k}^{\varepsilon}(\boldsymbol{x})=d_{3, k}^{\varepsilon}(\boldsymbol{x})-\varepsilon^{4} v^{(4)}(\boldsymbol{x})-\varepsilon^{4} V_{k}^{(3)}\left(\frac{\boldsymbol{x}}{\varepsilon}\right), \quad k \in\{f, m\} .
$$

The update of the difference allows us to obtain the fourth-order approximation:

$$
\begin{aligned}
u_{\varepsilon, k}^{[4]}(\boldsymbol{x})=u^{(0)}(\boldsymbol{x})+\varepsilon V_{k}^{(0)}\left(\frac{\boldsymbol{x}}{\varepsilon}\right)+\varepsilon^{2} V_{k}^{(1)}\left(\frac{\boldsymbol{x}}{\varepsilon}\right) & +\varepsilon^{2} v^{(2)}(\boldsymbol{x})+\varepsilon^{3} V_{k}^{(2)}\left(\frac{\boldsymbol{x}}{\varepsilon}\right)+\varepsilon^{3} v^{(3)}(\boldsymbol{x}) \\
& +\varepsilon^{4} v^{(4)}(\boldsymbol{x})+\varepsilon^{4} V_{k}^{(3)}\left(\frac{\boldsymbol{x}}{\varepsilon}\right), k \in\{f, m\} .
\end{aligned}
$$

Then, for $k \in\{f, m\}, d_{4, k}^{\varepsilon}$ solves the following problem:

$$
\begin{array}{rr}
\alpha_{f} \Delta d_{4, f}^{\varepsilon}(\boldsymbol{x})=0 & \text { in } \Omega_{f}^{\varepsilon,}, \\
\alpha_{m} \Delta d_{4, m}^{\varepsilon}(\boldsymbol{x})=0 & \text { in } \Omega_{m}^{\varepsilon}, \\
d_{4, f}^{\varepsilon}(\boldsymbol{x})=d_{4, m}^{\varepsilon}(\boldsymbol{x}) & \text { on } \Gamma^{\varepsilon}, \\
\alpha_{f} \partial_{\boldsymbol{n}} d_{4, f}^{\varepsilon}(\boldsymbol{x})=\alpha_{m} \partial_{\boldsymbol{n}} d_{4, m}^{\varepsilon}(\boldsymbol{x}) & \\
+\left(\alpha_{m}-\alpha_{f}\right)\left(\partial_{\boldsymbol{n}} u^{(0)}(\varepsilon \boldsymbol{y})-\omega_{0}^{(0)}(\boldsymbol{y})-\varepsilon \omega_{0}^{(1)}(\boldsymbol{y})-\varepsilon^{2} \omega_{0}^{(2)}-\varepsilon^{3} \omega_{0}^{(3)}(\boldsymbol{y})\right) & \\
+\varepsilon^{2}\left(\alpha_{m}-\alpha_{f}\right)\left(\partial_{\boldsymbol{n}} v^{(2)}(\varepsilon \boldsymbol{y})-\omega_{2}^{(0)}(\boldsymbol{y})-\varepsilon \omega_{2}^{(1)}(\boldsymbol{y})\right) & \\
+\varepsilon^{3}\left(\alpha_{m}-\alpha_{f}\right)\left(\partial_{\boldsymbol{n}} v^{(3)}(\varepsilon \boldsymbol{y})-\omega_{3}^{(0)}(\boldsymbol{y})\right)+\varepsilon^{4}\left(\alpha_{m}-\alpha_{f}\right)\left(\partial_{\boldsymbol{n}} v^{(4)}(\varepsilon \boldsymbol{y})\right) & \text { on } \Gamma^{\varepsilon}, \\
d_{4, m}^{\varepsilon}(\boldsymbol{x})=\varepsilon\left(\varepsilon^{3} \frac{a_{0}^{(3)}(\theta)}{|\boldsymbol{x}|^{3}}+\varepsilon^{2} \frac{a_{0}^{(2)}(\theta)}{|\boldsymbol{x}|^{2}}+\varepsilon \frac{a_{0}^{(1)}(\theta)}{|\boldsymbol{x}|}-V_{m}^{(0)}\left(\frac{\boldsymbol{x}}{\varepsilon}\right)\right) & \\
+\varepsilon^{2}\left(\varepsilon^{2} \frac{a_{1}^{(2)}(\theta)}{|\boldsymbol{x}|^{2}}+\varepsilon \frac{a_{1}^{(1)}(\theta)}{|\boldsymbol{x}|}-V_{m}^{(1)}\left(\frac{\boldsymbol{x}}{\varepsilon}\right)\right) & \\
+\varepsilon^{3}\left(\varepsilon \frac{a_{1}^{(1)}(\theta)}{|\boldsymbol{x}|}-V_{m}^{(2)}\left(\frac{\boldsymbol{x}}{\varepsilon}\right)\right)-\varepsilon^{4}\left(V_{m}^{(3)}\left(\frac{\boldsymbol{x}}{\varepsilon}\right)\right) & \text { on } \Gamma .
\end{array}
$$




\subsubsection{The $K$-th order approximation}

From the previous section we are able to deduce the general forms of $\varphi^{(n)}$ and $\psi^{(n)}$ so we can update our residual to the order $n \geq 2$ as follows:

$$
d_{n, k}^{\varepsilon}(\boldsymbol{x})=d_{n-1, k}^{\varepsilon}(\boldsymbol{x})-\varepsilon^{n} v^{(n)}(\boldsymbol{x})-\varepsilon^{n} V_{k}^{(n-1)}\left(\frac{\boldsymbol{x}}{\varepsilon}\right), \quad k \in\{f, m\},
$$

where $V^{(n-1)}$ solves Problem (3.5) for $\varphi^{(n-1)}(\boldsymbol{y})=\left(\alpha_{m}-\alpha_{f}\right) \sum_{\substack{p+q=n-1 \\ p \geq 0, q \neq 1}} \boldsymbol{\omega}_{q}^{(p)}(\boldsymbol{y}) \cdot \boldsymbol{n}$ and $v^{(n)}$ solves Problem (3.10) for $\psi^{(n)}(\boldsymbol{x})=-\sum_{\substack{p+q=n-1 \\ p \geq 1, q \geq 0}} \frac{a_{q}^{(p)}(\theta)}{|\boldsymbol{x}|^{p}}$.

We are now able to build an approximation $u_{\varepsilon}^{[K]}(\boldsymbol{x})$ to the order $K \geq 2$ :

$$
u_{\varepsilon, k}^{[K]}(\boldsymbol{x})=u^{(0)}(\boldsymbol{x})+\sum_{i=2}^{K} \varepsilon^{i} v^{(i)}(\boldsymbol{x})+\sum_{i=1}^{K} \varepsilon^{i} V_{k}^{(i-1)}\left(\frac{\boldsymbol{x}}{\varepsilon}\right), \quad k \in\{f, m\} .
$$

Remark 3.2. According to the power of $\varepsilon$ this is not the expansion to the order $K$ in the true sense. Indeed, it is the expansion to the order $K+1$ truncated of a term. To clearly see this, let us take the example where $K$ is equal to 1 . By analyzing the expression of $u_{\varepsilon}^{[1]}(\boldsymbol{x})$, we notice that initially the expansion has a term in $\varepsilon^{2}\left(\varepsilon V^{(0)}\left(\frac{\boldsymbol{x}}{\varepsilon}\right)\right)$. However, an $\varepsilon^{2}$ order term will appear at the second order $\left(\varepsilon^{2} v^{(2)}\right)$. So, in order to obtain all the terms in $\varepsilon^{K}$, we have to push the expansion at the order $K$ and truncate the term $\varepsilon^{K} V^{K}$.

Now, replacing $u_{\varepsilon}^{[K]}(\boldsymbol{x})$ in Problem (2.1) we obtain the following problem:

$$
\begin{array}{rc}
\alpha_{f} \Delta u_{\varepsilon}^{[K]}(\boldsymbol{x})=0 & \text { in } \Omega_{f}^{\varepsilon}, \\
\alpha_{m} \Delta u_{\varepsilon}^{[K]}(\boldsymbol{x})=0 & \text { in } \Omega_{m}^{\varepsilon}, \\
\llbracket u_{\varepsilon}^{[K]}(\boldsymbol{x}) \rrbracket=0 & \text { on } \Gamma^{\varepsilon}, \\
\llbracket \alpha \partial_{\boldsymbol{n}} u_{\varepsilon}^{[K]}(\boldsymbol{x}) \rrbracket=g_{\varepsilon}(\boldsymbol{y}) & \text { on } \Gamma^{\varepsilon}, \\
u_{\varepsilon}^{[K]}(\boldsymbol{x})=h_{\varepsilon}(\boldsymbol{x})+F & \text { on } \Gamma,
\end{array}
$$

where

$$
g_{\varepsilon}(\boldsymbol{y})=\left(\alpha_{m}-\alpha_{f}\right) \partial_{\boldsymbol{n}} u^{(0)}(\varepsilon \boldsymbol{y})+\sum_{i=2}^{K} \varepsilon^{i}\left(\alpha_{m}-\alpha_{f}\right) \partial_{\boldsymbol{n}} v^{(i)}(\varepsilon \boldsymbol{y})+\sum_{i=1}^{K} \varepsilon^{i-1} \llbracket \alpha \partial_{\boldsymbol{n}} V^{(i-1)}(\boldsymbol{y}) \rrbracket_{\Gamma_{1}} .
$$

Using the expressions of $\varphi^{(i-1)}(\boldsymbol{y})$ we obtain the expression of the jump $\llbracket \alpha \partial_{\boldsymbol{n}} V^{(i-1)}(\boldsymbol{y}) \rrbracket_{\Gamma_{1}}=$ $\left(\alpha_{f}-\alpha_{m}\right) \sum_{\substack{p+q=i-1 \\ p \geq 0, q \neq 1}} \boldsymbol{\omega}_{q}^{(p)}(\boldsymbol{y}) \cdot \boldsymbol{n}:$

$$
\begin{aligned}
g_{\varepsilon}(\boldsymbol{y})=\left(\alpha_{m}-\alpha_{f}\right) & \partial_{\boldsymbol{n}} u^{(0)}(\varepsilon \boldsymbol{y})+\sum_{i=2}^{K} \varepsilon^{i}\left(\alpha_{m}-\alpha_{f}\right) \partial_{\boldsymbol{n}} v^{(i)}(\varepsilon \boldsymbol{y}) \\
& +\sum_{i=1}^{K} \varepsilon^{i-1}\left[\left(\alpha_{f}-\alpha_{m}\right) \sum_{\substack{p+q=i-1 \\
p \geq 0, q \neq 1}} \boldsymbol{\omega}_{q}^{(p)}(\boldsymbol{y}) \cdot \boldsymbol{n}\right]+O\left(\varepsilon^{K+1}\right)=\varepsilon^{K} f(\boldsymbol{y}) .
\end{aligned}
$$

The last step is to evaluate the $H^{-\frac{1}{2}}\left(\Gamma^{\varepsilon}\right)$-norm of $\varepsilon^{K} f(\boldsymbol{y})$ on $\Gamma^{\varepsilon}$ :

$\left\|\varepsilon^{K} f\left(\frac{\boldsymbol{x}}{\varepsilon}\right)\right\|_{H^{-\frac{1}{2}}\left(\Gamma^{\varepsilon}\right)}^{2}=\int_{\Gamma^{\varepsilon}} \int_{\Gamma^{\varepsilon}} \varepsilon^{2 K}\left|f\left(\frac{x_{1}}{\varepsilon}\right)-f\left(\frac{x_{2}}{\varepsilon}\right)\right| \mathrm{d} \Gamma^{\varepsilon} \mathrm{d} \Gamma^{\varepsilon}=\int_{\Gamma^{1}} \int_{\Gamma^{1}} \varepsilon^{2 K+2}\left|f\left(y_{1}\right)-f\left(y_{2}\right)\right| \mathrm{d} \Gamma^{1} \mathrm{~d} \Gamma^{1}=C \varepsilon^{2 K+2}$. 
Finally, we obtain:

$$
\left\|g_{\varepsilon}(\boldsymbol{y})\right\|_{H^{-\frac{1}{2}}\left(\Gamma^{\varepsilon}\right)}=\left\|\varepsilon^{K} f\left(\frac{\boldsymbol{x}}{\varepsilon}\right)\right\|_{H^{-\frac{1}{2}\left(\Gamma^{\varepsilon}\right)}}=O\left(\varepsilon^{K+1}\right) .
$$

By applying exactly the same approach we get:

$$
\left\|h_{\varepsilon}(\boldsymbol{x})\right\|_{H^{s}(\Gamma)}=O\left(\varepsilon^{K+1}\right) .
$$

Consequently, $d_{K, k}^{\varepsilon}=u_{k}^{\varepsilon}-u_{\varepsilon, k}^{[K]}(\boldsymbol{x}), k \in\{f, m\}$ satisfy:

$$
\begin{aligned}
\alpha_{f} \Delta d_{K, f}^{\varepsilon}(\boldsymbol{x})=0 & \text { in } \Omega_{f}^{\varepsilon}, \\
\alpha_{m} \Delta d_{K, m}^{\varepsilon}(\boldsymbol{x})=0 & \text { in } \Omega_{m}^{\varepsilon}, \\
d_{K, f}^{\varepsilon}(\boldsymbol{x})=d_{K, m}^{\varepsilon}(\boldsymbol{x}) & \text { on } \Gamma^{\varepsilon}, \\
\alpha_{f} \partial_{\boldsymbol{n}} d_{K, f}^{\varepsilon}(\boldsymbol{x})=\alpha_{m} \partial_{\boldsymbol{n}} d_{K, m}^{\varepsilon}(\boldsymbol{x})+O_{H^{-\frac{1}{2}\left(\Gamma^{\varepsilon}\right)}}\left(\varepsilon^{K+1}\right) & \text { on } \Gamma^{\varepsilon}, \\
d_{K, m}^{\varepsilon}(\boldsymbol{x})=O_{H^{\frac{1}{2}(\Gamma)}}\left(\varepsilon^{K+1}\right) & \text { on } \Gamma .
\end{aligned}
$$

\subsection{Existence and uniqueness in unbounded domains for the Poisson problem}

A weak formulation of Problem (3.5) writes:

$$
\alpha_{f} \int_{\Omega_{f}^{1}} \nabla V_{f}^{(p)} \cdot \nabla w \mathrm{~d} \boldsymbol{x}+\alpha_{m} \int_{\Omega^{\infty}} \nabla V_{m}^{(p)} \cdot \nabla w \mathrm{~d} \boldsymbol{x}=\int_{\Gamma^{1}} \varphi w \mathrm{~d} \sigma, \quad \forall w \in \mathcal{V},
$$

where $\mathcal{V}=\left\{v \in \mathcal{W}_{0,0}^{1,2} ; \quad \int_{\Gamma_{R_{0}}} v=0\right\}$ with $0<R_{0}<R$ an arbitrary real number and $\mathcal{W}_{0,0}^{1,2}$ is introduced in the appendix. In fact, the space $\mathcal{W}_{0,0}^{1,2}$ contains constant functions and the Poincarétype inequality is established on $\mathcal{W}_{0,0}^{1,2} / P^{0}$, the quotient space of the adequate weighted Sobolev space with constant functions. We can see that the bilinear form $a(z, w)=\alpha_{f} \int_{\Omega_{f}^{1}} \nabla z \cdot \nabla w \mathrm{~d} \boldsymbol{x}+$ $\alpha_{m} \int_{\Omega^{\infty}} \nabla z \cdot \nabla w \mathrm{~d} \boldsymbol{x}$ is coercive on $\mathcal{V}$. In addition, the bilinear form $a(\cdot, \cdot)$ and the linear one $l(w)=\int_{\Gamma^{1}} \varphi w \mathrm{~d} \sigma$ are continuous on $\mathcal{V}$. Then, the existence and uniqueness of the functions $V^{(p)}$ in $\mathcal{V}$ are guaranteed by the Lax-Milgram theorem.

\subsection{Expansion of the functions $V_{m}^{(p)}$ at infinity}

Recall that the functions $V_{m}^{(p)}$ solve Problem (3.5). Let $B(O, R)$ be the ball of center $O$ and radius $R>0$ such that $\Omega_{f}^{1} \subset B(O, R)$. Then, the trace of $V_{m}^{(p)}$ on the curve $\partial B(O, R) \cap \Omega^{\infty}$ is smooth and can be written as the sum of its Fourier series:

$$
V_{m}^{(p)}(R, \theta)=\alpha_{0}^{(p)}+\sum_{n \geq 1} \alpha_{n}^{(p)} \cos (n \theta)+\beta_{n}^{(p)} \sin (n \theta) .
$$

Using Poisson's Kernel, we then get that:

$$
V_{m}^{(p)}(r, \theta)=\alpha_{0}^{(p)}+\sum_{n \geq 1} \frac{R^{n}}{r^{n}}\left(\alpha_{n}^{(p)} \cos (n \theta)+\beta_{n}^{(p)} \sin (n \theta)\right) .
$$

The behavior of the function $V_{m}^{(p)}$ at infinity prescribes $\alpha_{0}^{(p)}=0$. 


\subsection{Error estimate for the antiplane problem}

In this section we establish an error estimate for the studied problem by increasing the $H^{1}$ norm by powers of $\varepsilon$ (characteristic length of the inclusion). First, we begin by considering the following problem for a given $g \in H^{-\frac{1}{2}}\left(\Gamma^{\varepsilon}\right)$ and $h \in H^{\frac{1}{2}}(\Gamma)$ :

$$
\begin{aligned}
\alpha_{f} \Delta_{\boldsymbol{x}} z_{f}(\boldsymbol{x})=0 & \text { in } \Omega_{f}^{\varepsilon}, \\
\alpha_{m} \Delta_{\boldsymbol{x}} z_{m}(\boldsymbol{x})=0 & \text { in } \Omega_{m}^{\varepsilon}, \\
z_{f}(\boldsymbol{x})=z_{m}(\boldsymbol{x}) & \text { on } \Gamma^{\varepsilon}, \\
\alpha_{f} \partial_{\boldsymbol{n}} z_{f}(\boldsymbol{x})=\alpha_{m} \partial_{\boldsymbol{n}} z_{m}(\boldsymbol{x})+g(\boldsymbol{x}) & \text { on } \Gamma^{\varepsilon}, \\
z_{m}(\boldsymbol{x})=h(\boldsymbol{x}) & \text { on } \Gamma .
\end{aligned}
$$

By passing to a weak form we obtain the following equivalent problem: Find $z \in H^{1}(\Omega)$ with $z=h$ on $\Gamma$ such that

$$
\int_{\Omega_{f}^{\varepsilon}} \nabla z \cdot \nabla w \mathrm{~d} \boldsymbol{x}+\int_{\Omega_{m}^{\varepsilon}} \nabla z \cdot \nabla w \mathrm{~d} \boldsymbol{x}+\int_{\Gamma^{\varepsilon}} g w \mathrm{~d} \sigma=0, \quad \forall \omega \in H_{0}^{1}(\Omega) .
$$

Lemma 3.3. There exists a unique solution $z$ of the weak form (3.36) of Problem (3.35) satisfying

$$
\|z\|_{H^{1}(\Omega)} \leq C\left(\|h\|_{H^{\frac{1}{2}(\Gamma)}}+\|g\|_{H^{-\frac{1}{2}\left(\Gamma^{\varepsilon}\right)}}\right),
$$

where $C>0$ is a constant independent of $\varepsilon$.

Proof. The surjectivity of the trace operator guarantees the existence of a continuous lifting operator $H \in H^{1}(\Omega)$ of $h \in H^{\frac{1}{2}}(\Gamma)$ (see e.g. [28, 29]): Then $\bar{z}=z-H \in H_{0}^{1}(\Omega)$ solves the following variational equation:

$$
\int_{\Omega_{f}^{\varepsilon}} \nabla \bar{z} \cdot \nabla w \mathrm{~d} \boldsymbol{x}+\int_{\Omega_{m}^{\varepsilon}} \nabla \bar{z} \cdot \nabla w \mathrm{~d} \boldsymbol{x}+\int_{\Gamma^{\varepsilon}} g w \mathrm{~d} \sigma+\int_{\Omega} \nabla H \cdot \nabla w \mathrm{~d} \boldsymbol{x}=0, \quad \forall w \in H_{0}^{1}(\Omega) .
$$

The bilinear form associated to this weak formulation $a(u, v)=\int_{\Omega_{f}^{\varepsilon}} \nabla u \cdot \nabla v \mathrm{~d} \boldsymbol{x}+\int_{\Omega_{m}^{\varepsilon}} \nabla u \cdot \nabla v \mathrm{~d} \boldsymbol{x}$ is coercive, i.e. $\alpha\|v\|_{H^{1}(\Omega)}^{2} \leq a(v, v)$, see [28,29]. Then, from the Lax-Milgram theorem we deduce the existence and uniqueness of $\bar{z}$ solution of (3.37). The coercivity of this bilinear form yields:

$$
\alpha\|\bar{z}\|_{H^{1}(\Omega)}^{2} \leq-\int_{\Gamma^{\varepsilon}} g \bar{z} \mathrm{~d} \sigma-\int_{\Omega} \nabla H \cdot \nabla \bar{z} \mathrm{~d} \boldsymbol{x},
$$

and hence

$$
\alpha\|\bar{z}\|_{H^{1}(\Omega)}^{2} \leq\|g\|_{H^{-\frac{1}{2}\left(\Gamma^{\varepsilon}\right)}}\|\bar{z}\|_{H^{\frac{1}{2}\left(\Gamma^{\varepsilon}\right)}}+\|\nabla H\|_{L^{2}(\Omega)}\|\nabla \bar{z}\|_{L^{2}(\Omega)} .
$$

Using the facts that $\|\bar{z}\|_{H^{\frac{1}{2}\left(\Gamma^{\varepsilon}\right)}} \leq C\|\bar{z}\|_{H^{1}(\Omega)},\|\nabla \bar{z}\|_{L^{2}(\Omega)} \leq C\|\bar{z}\|_{H^{1}(\Omega)}$ and $\|\nabla H\|_{L^{2}(\Omega)} \leq$ $C\|H\|_{H^{1}(\Omega)}$ we obtain:

$$
\alpha\|\bar{z}\|_{H^{1}(\Omega)}^{2} \leq C\left(\|g\|_{H^{-\frac{1}{2}\left(\Gamma^{\varepsilon}\right)}}+\|H\|_{H^{1}(\Omega)}\right)\|\bar{z}\|_{H^{1}(\Omega)} .
$$

The continuity of the lifting operator gives us that $\|H\|_{H^{1}(\Omega)} \leq C\|h\|_{H^{\frac{1}{2}(\Gamma)}}$, so we can write:

$$
\|\bar{z}\|_{H^{1}(\Omega)} \leq C\left(\|g\|_{H^{-\frac{1}{2}\left(\Gamma^{\varepsilon}\right)}}+\|h\|_{H^{\frac{1}{2}(\Gamma)}}\right) .
$$


We are now ready to state the following result:

Theorem 3.4. There exists a constant $C>0$ independent of $\varepsilon$ such that:

$$
\left\|d_{K}^{\varepsilon}\right\|_{H^{1}(\Omega)} \leq C \varepsilon^{K+1}, \quad \text { for all } K \in \mathbb{N},
$$

where $d_{K}^{\varepsilon}$ is the residual introduced in Section 3.

Proof. With $z=d_{K}^{\varepsilon}$ we have $g \in O_{H^{-\frac{1}{2}}\left(\Gamma^{\varepsilon}\right)}\left(\varepsilon^{K+1}\right)$ and $h \in O_{H^{\frac{1}{2}}(\Gamma)}\left(\varepsilon^{K+1}\right)$ (see Section 3.1.5).

\subsection{Solution for a circular inclusion using the separation of variable method}

In this section, we solve Problem (2.1) by the separation of variable method then we solve Problem (3.5) by the same method and compare the two results for the first-order of the asymptotic expansion. We will use the same geometrical configuration but with a circular shape for the interface $\Gamma^{\varepsilon}$ and the boundary $\Gamma^{1}$ (see Fig. 3).

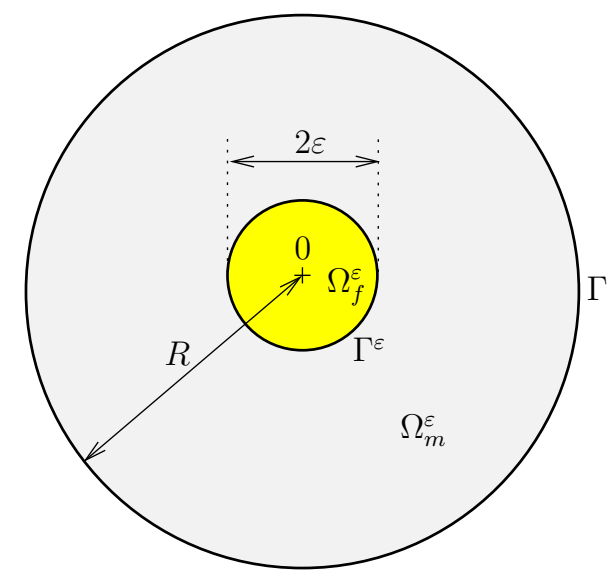

Figure 3: Circular inclusion and domain.

\subsubsection{Antiplane problem solve using the separation of variable method}

Considering the polar coordinates $(r, \theta)$ and the following particular form for the $u_{k}^{\varepsilon}, k \in\{f, m\}$ :

$$
u_{k}^{\varepsilon}(r, \theta)=f_{k}(r) g_{k}(\theta)
$$

we obtain from equations (2.1a) and (2.1b) the following equations:

$$
r^{2} \frac{f_{k}^{\prime \prime}(r)}{f_{k}(r)}+r \frac{f_{k}^{\prime}(r)}{f_{k}(r)}=-\frac{g_{k}^{\prime \prime}(\theta)}{g(\theta)}, \quad k \in\{f, m\} .
$$

The left hand sides of these equations are independent of $\theta$ and the right ones are independent of $r$. Then they are both equal to the same constant, called separation constant, that can be proven to be equal to $n_{k}^{2}$ with $n_{k} \in \mathbb{N}$. This leads to the system of two decoupled equations:

$$
\begin{aligned}
r^{2} f_{k}^{\prime \prime}(r)+r f_{k}^{\prime}(r)-n_{k}^{2} f_{k}(r) & =0 \\
g_{k}^{\prime \prime}(\theta)+n_{k}^{2} g_{k}(\theta) & =0 .
\end{aligned}
$$


Using the equation (2.1c) we can see that $g_{1}(\theta)=g_{2}(\theta)$ and then $n_{k}=n$. We observe now that we can search solutions of the forms of $r^{\alpha}$ and thus we obtain:

$$
\begin{aligned}
f_{f}(r) & =C_{1} r^{n}+c r^{-n} \\
f_{m}(r) & =C_{2} r^{n}+C_{3} r^{-n} .
\end{aligned}
$$

The solution must be bounded at the origin, hence $c=0$. The next step is to use the continuity of the fields and their normal derivatives throughout the interface $\Gamma^{\varepsilon}$ to determine the constants $C_{1}, C_{2}$ and $C_{3}$. In fact, from equations (2.1c) and (2.1d) we obtain with $\beta=\frac{\alpha_{m}-\alpha_{f}}{\alpha_{m}+\alpha_{f}}$ and $h=\frac{\varepsilon}{R}$ the following expressions:

$$
\begin{aligned}
& C_{1}(n)=\frac{R^{-n}(1+\beta)}{1+\beta h^{2 n}}, \\
& C_{2}(n)=\frac{R^{-n}}{1+\beta h^{2 n}}, \\
& C_{3}(n)=\frac{R^{n} \beta h^{2 n}}{1+\beta h^{2 n}} .
\end{aligned}
$$

By expanding $C_{1}, C_{2}$ and $C_{3}$ with respect to the small parameter $h$ and taking $n=1$ we can bring up the first contribution of the inclusion in the expression of matrix field:

$$
u_{m}^{\varepsilon}=u_{m}^{(0)}+h u_{m}^{(1)}+h^{2} u_{m}^{(2)} .
$$

Since $u_{m}^{(1)}$ is zero and $u_{m}^{(0)}, u_{m}^{(2)}$ are independent of $\varepsilon$ so we can conclude that the first contribution of the inclusion is of order $\varepsilon^{2}$.

\subsubsection{Solving $V^{(0)}$ by the separation of variable method}

Thanks to the separation of variable method and using polar coordinates $\left(r_{s}, \theta\right)\left(r_{s}=\frac{r}{\varepsilon}\right)$ we decompose the functions $V_{k}^{(0)}$ as follows:

$$
V_{k}^{(0)}\left(r_{s}, \theta\right)=\sum_{n \geq 1} f_{n}^{(k)}\left(r_{s}\right) \cos (n \theta)+g_{n}^{(k)}\left(r_{s}\right) \sin (n \theta), \quad k \in\{f, m\} .
$$

Injecting (3.48) in (3.5a) and (3.5b) and solving the differential equations we obtain:

$$
\begin{aligned}
f_{n}^{k} & =A_{k} r_{s}^{n}+a_{k} r_{s}^{-n}, \\
g_{n}^{k} & =B_{k} r_{s}^{n}+b_{k} r_{s}^{-n} .
\end{aligned}
$$

Using the interface conditions prescribed by $(3.5 \mathrm{c})$ and $(3.5 \mathrm{~d})$ we obtain:

$$
V_{m}^{(0)}\left(r_{s}, \theta\right)=\frac{\alpha_{m}-\alpha_{f}}{r_{s}}\left(\frac{a}{\alpha_{f}+\alpha_{m}} \cos \theta+\frac{b}{\alpha_{f}+\alpha_{m}} \sin \theta\right) .
$$

with $\boldsymbol{\nabla} u^{(0)}(\mathbf{0})=(a b)^{t}$. Thus, Solving directly Problem (2.1) by the separation of variable method we obtain that the first contribution of the inclusion is in $\varepsilon^{2}$. Now injecting (3.50) in (3.8) we obtain:

$$
u_{\varepsilon, m}^{[1]}(r, \theta)=u^{(0)}+\frac{\varepsilon^{2}}{r}\left(\frac{\left(\alpha_{m}-\alpha_{f}\right) a}{\alpha_{f}+\alpha_{m}} \cos \theta+\frac{\left(\alpha_{m}-\alpha_{f}\right) b}{\alpha_{f}+\alpha_{m}} \sin \theta\right) .
$$

The first contribution is indeed in $\varepsilon^{2}$ which is in accordance with the result presented in Section 3.5.1. 


\section{Asymptotic expansion for the plane strain linear elasticity prob- lem}

We now propose an adaptation of the construction of the asymptotic expansion to the vector valued case of the plain strain elasticity problem (2.2). One of the main differences will be the appearance of an additional term in the expansion related to the difference of the constitutive laws in the inclusion and in the matrix. The form of expansion is as follows:

$$
\boldsymbol{u}_{\varepsilon, k}^{[K]}(\boldsymbol{x})=\boldsymbol{u}^{(0)}(\boldsymbol{x})+\sum_{i=2}^{K} \varepsilon^{i} \boldsymbol{v}^{(i)}(\boldsymbol{x})+\sum_{i=1}^{K} \varepsilon^{i} \boldsymbol{V}_{k}^{(i-1)}\left(\frac{\boldsymbol{x}}{\varepsilon}\right)+\sum_{i=1}^{K} \varepsilon^{i+1} \boldsymbol{Z}_{k}^{(i-1)}\left(\frac{\boldsymbol{x}}{\varepsilon}\right)
$$

where $\boldsymbol{u}^{(0)}$ denotes the solution to Problem (2.2) still for $\varepsilon=0$, the functions $\boldsymbol{v}^{(i)}$ are defined on $\Omega$ and the $\boldsymbol{V}_{k}^{(i)}$ and $\boldsymbol{Z}_{k}^{(i)}$ functions on the whole $\mathbb{R}^{2}$.

The structure of this section globally follows the one for the Poisson problem in Section 3 apart from the fact that we do not propose a resolution by separation of variables, mainly because it gives no easily exploitable result in that case. Of course, an approximation by boundary elements or a Galerkin method can still be considered. The result on the behavior at infinity of functions $\boldsymbol{V}^{(i)}$ and $\boldsymbol{Z}^{(i)}$ is given in section 4.3 but only in the isotropic case. This also limits to the isotropic case the error estimate given in Section 4.4.

\subsection{Building the asymptotic expansion}

The difference $\boldsymbol{d}_{0, k}^{\varepsilon}=\boldsymbol{u}_{k}^{\varepsilon}-\boldsymbol{u}^{(0)}(k \in\{f, m\})$ is now solution to:

$$
\begin{array}{rc}
\operatorname{div}{ }_{\boldsymbol{x}} \boldsymbol{\sigma}_{f}\left(\boldsymbol{d}_{0, f}^{\varepsilon}\right)(\boldsymbol{x})=-\operatorname{div}_{\boldsymbol{x}}\left(\boldsymbol{E}_{f}: \boldsymbol{\epsilon}\left(\boldsymbol{u}^{(0)}\right)\right)(\boldsymbol{x}) & \text { in } \Omega_{f}^{\varepsilon}, \\
\operatorname{div}_{\boldsymbol{x}} \boldsymbol{\sigma}_{m}\left(\boldsymbol{d}_{0, m}^{\varepsilon}(\boldsymbol{x})\right)=\mathbf{0} & \text { in } \Omega_{m}^{\varepsilon}, \\
\boldsymbol{d}_{0, f}^{\varepsilon}(\boldsymbol{x})=\boldsymbol{d}_{0, m}^{\varepsilon}(\boldsymbol{x}) & \text { on } \Gamma^{\varepsilon}, \\
\boldsymbol{\sigma}_{f}\left(\boldsymbol{d}_{0, f}^{\varepsilon}(\boldsymbol{x})\right) \cdot \boldsymbol{n}_{f}=-\boldsymbol{\sigma}_{m}\left(\boldsymbol{d}_{0, m}^{\varepsilon}(\boldsymbol{x})\right) \cdot \boldsymbol{n}_{m}+\left[\left(\boldsymbol{E}_{m}-\boldsymbol{E}_{f}\right): \boldsymbol{\epsilon}\left(\boldsymbol{u}^{(0)}(\boldsymbol{x})\right)\right] \cdot \boldsymbol{n}_{f} & \text { on } \Gamma^{\varepsilon}, \\
\boldsymbol{d}_{0, m}^{\varepsilon}(\boldsymbol{x})=\mathbf{0} & \text { on } \Gamma .
\end{array}
$$

This time, we note the emergence of two terms $\left[\left(\boldsymbol{E}_{m}-\boldsymbol{E}_{f}\right): \boldsymbol{\epsilon}\left(\boldsymbol{u}^{0}\right)(\boldsymbol{x})\right] \cdot \boldsymbol{n}_{f}$ on $\Gamma^{\varepsilon}$, which is similar to the term $\left(\alpha_{m}-\alpha_{f}\right) \partial_{\boldsymbol{n}} u^{(0)}(\boldsymbol{x})$ of the antiplane problem, and a new term $-\operatorname{div}\left(\boldsymbol{E}_{f}: \boldsymbol{\epsilon}\left(\boldsymbol{u}^{(0)}\right)\right)(\boldsymbol{x})$ in $\Omega_{f}^{\varepsilon}$.

Still using the scaling $\boldsymbol{y}=\frac{\boldsymbol{x}}{\varepsilon}$ and denoting $\Omega^{m, \varepsilon}=\frac{\Omega_{m}^{\varepsilon}}{\varepsilon}, \boldsymbol{D}_{0, k}(\boldsymbol{y})=\boldsymbol{d}_{0, k}(\boldsymbol{x})(k \in\{f, m\})$ is solution to the following scaled problem:

$$
\begin{array}{rc}
\left.\operatorname{div} \boldsymbol{y}_{f}\left(\boldsymbol{D}_{0, f}^{\varepsilon}\right)(\boldsymbol{y})=-\varepsilon^{2} \operatorname{div}{ }_{\boldsymbol{x}}\left(\boldsymbol{E}_{f}: \boldsymbol{\epsilon}\left(\boldsymbol{u}^{(0)}\right)\right)(\varepsilon \boldsymbol{y})\right) & \text { in } \Omega_{f}^{1}, \\
\operatorname{div} \boldsymbol{y}_{m}\left(\boldsymbol{D}_{0, m}^{\varepsilon}\right)(\boldsymbol{y})=\mathbf{0} & \text { in } \Omega^{m, \varepsilon}, \\
\boldsymbol{D}_{0, f}^{\varepsilon}(\boldsymbol{y})=\boldsymbol{D}_{0, m}^{\varepsilon}(\boldsymbol{y}) & \text { on } \Gamma^{1}, \\
\boldsymbol{\sigma}_{f}\left(\boldsymbol{D}_{0, f}^{\varepsilon}\right)(\boldsymbol{y}) \cdot \boldsymbol{n}_{f}=-\boldsymbol{\sigma}_{m}\left(\boldsymbol{D}_{0, m}^{\varepsilon}\right)(\boldsymbol{y}) \cdot \boldsymbol{n}_{m} & \\
+\varepsilon\left[\left(\boldsymbol{E}_{m}-\boldsymbol{E}_{f}\right): \boldsymbol{\epsilon}_{\boldsymbol{x}}\left(\boldsymbol{u}^{(0)}(\varepsilon \boldsymbol{y})\right)\right] \cdot \boldsymbol{n}_{f} & \text { on } \Gamma^{1}, \\
\boldsymbol{D}_{0, m}^{\varepsilon}(\boldsymbol{y})=\mathbf{0} & \text { on } \frac{\Gamma}{\varepsilon} .
\end{array}
$$


Now, as $\varepsilon$ is considered to be small compared to the size of the domain, we approximate Problem (4.3) by the following problem defined on the dilated domain $\Omega^{\infty}=\lim _{\varepsilon \rightarrow 0} \Omega^{m, \varepsilon}$ :

$$
\begin{aligned}
\left.\operatorname{div} \boldsymbol{\sigma}_{1}\left(\boldsymbol{D}_{0, f}^{\varepsilon}\right)(\boldsymbol{y})=-\varepsilon^{2} \operatorname{div}{ }_{\boldsymbol{x}}\left(\boldsymbol{E}_{f}: \boldsymbol{\epsilon}\left(\boldsymbol{u}^{(0)}\right)\right)(\varepsilon \boldsymbol{y})\right) & \text { in } \Omega_{f}^{1}, \\
\operatorname{div} \boldsymbol{y}_{m}\left(\boldsymbol{D}_{0, m}^{\varepsilon}\right)(\boldsymbol{y})=\mathbf{0} & \text { in } \Omega^{\infty}, \\
\boldsymbol{D}_{0, f}^{\varepsilon}(\boldsymbol{y})=\boldsymbol{D}_{0, m}^{\varepsilon}(\boldsymbol{y}) & \text { on } \Gamma^{1}, \\
\boldsymbol{\sigma}_{f}\left(\boldsymbol{D}_{0, f}^{\varepsilon}\right)(\boldsymbol{y}) \cdot \boldsymbol{n}_{f}=-\boldsymbol{\sigma}_{m}\left(\boldsymbol{D}_{0, m}^{\varepsilon}\right)(\boldsymbol{y}) \cdot \boldsymbol{n}_{m} & \\
+\varepsilon\left[\left(\boldsymbol{E}_{m}-\boldsymbol{E}_{f}\right): \boldsymbol{\epsilon}_{\boldsymbol{x}}\left(\boldsymbol{u}^{(0)}(\varepsilon \boldsymbol{y})\right)\right] \cdot \boldsymbol{n}_{f} & \text { on } \Gamma^{1}, \\
\boldsymbol{D}_{0, m}^{\varepsilon}(\boldsymbol{y}) \rightarrow \mathbf{0} & \text { when }|\boldsymbol{y}| \rightarrow \infty .
\end{aligned}
$$

Let us then introduce two problems which will allow us to construct the different orders of the asymptotic expansion for the contribution of the inclusion. Both of these problems are defined on the dilated domain $\Omega^{\infty}$. For each order $p$, we consider the functions $\boldsymbol{V}^{(p)}$ and $\boldsymbol{Z}^{(p)}$ solutions to:

$$
\begin{aligned}
\operatorname{div}{ }_{\boldsymbol{y}} \boldsymbol{\sigma}_{f}\left(\boldsymbol{V}_{f}^{(p)}\right)(\boldsymbol{y})=\mathbf{0} & \text { in } \Omega_{f}^{1}, \\
\operatorname{div} \boldsymbol{y}_{m}\left(\boldsymbol{V}_{m}^{(p)}\right)(\boldsymbol{y})=\mathbf{0} & \text { in } \Omega^{\infty}, \\
\boldsymbol{V}_{f}^{(p)}(\boldsymbol{y})=\boldsymbol{V}_{m}^{(p)}(\boldsymbol{y}) & \text { on } \Gamma^{1}, \\
\boldsymbol{\sigma}_{f}\left(\boldsymbol{V}_{f}^{(p)}\right)(\boldsymbol{y}) \cdot \boldsymbol{n}_{f}=-\boldsymbol{\sigma}_{m}\left(\boldsymbol{V}_{m}^{(p)}\right)(\boldsymbol{y}) \cdot \boldsymbol{n}_{m}+\boldsymbol{\varphi}^{(p)}(\boldsymbol{y}) & \text { on } \Gamma^{1}, \\
\boldsymbol{V}_{m}^{(p)}(\boldsymbol{y}) \rightarrow \mathbf{0} & \text { when }|\boldsymbol{y}| \rightarrow \infty,
\end{aligned}
$$

and

$$
\begin{aligned}
\operatorname{div} \boldsymbol{y}_{f} \boldsymbol{\sigma}_{f}\left(\boldsymbol{Z}_{f}^{(p)}\right)(\boldsymbol{y})=\boldsymbol{\kappa}^{(p)}(\boldsymbol{y}) & \text { in } \Omega_{f}^{1}, \\
\operatorname{div} \boldsymbol{y}_{m}\left(\boldsymbol{Z}_{m}^{(p)}\right)(\boldsymbol{y})=\mathbf{0} & \text { in } \Omega^{\infty}, \\
\boldsymbol{Z}_{f}^{(p)}(\boldsymbol{y})=\boldsymbol{Z}_{m}^{(p)}(\boldsymbol{y}) & \text { on } \Gamma^{1}, \\
\boldsymbol{\sigma}_{f}\left(\boldsymbol{Z}_{f}^{(p)}\right)(\boldsymbol{y}) \cdot \boldsymbol{n}_{f}=-\boldsymbol{\sigma}_{m}\left(\boldsymbol{Z}_{m}^{(p)}\right)(\boldsymbol{y}) \cdot \boldsymbol{n}_{m} & \text { on } \Gamma^{1}, \\
\boldsymbol{Z}_{m}^{(p)}(\boldsymbol{y}) \rightarrow \mathbf{0} & \text { when }|\boldsymbol{y}| \rightarrow \infty .
\end{aligned}
$$

The functions $\boldsymbol{V}^{(p)}$ and $\boldsymbol{Z}^{(p)}$ are introduced to offset terms appearing on the boundary $\Gamma^{\varepsilon}$ and in the domain $\Omega_{f}^{\varepsilon}$ where the data $\boldsymbol{\varphi}^{(p)}(\boldsymbol{y})$ and $\boldsymbol{\kappa}^{(p)}(\boldsymbol{y})$ will be adapted to each order $p$. The existence and uniqueness of the functions $\boldsymbol{V}^{(p)}$ and $\boldsymbol{Z}^{(p)}$ are established later in Section 4.2. From the regularity assumptions on the domain we can write Taylor expansions of $\boldsymbol{\epsilon}_{\boldsymbol{x}}\left(\boldsymbol{u}^{(0)}\right)(\varepsilon \boldsymbol{y})$ for $\boldsymbol{y} \in \Gamma^{1}$ and $-\operatorname{div}_{\boldsymbol{x}}\left(\boldsymbol{E}_{f}: \boldsymbol{\epsilon}\left(\boldsymbol{u}^{(0)}\right)\right)(\varepsilon \boldsymbol{y})$ for $\boldsymbol{y} \in \Omega_{f}^{1}$ :

$$
\boldsymbol{\epsilon}_{\boldsymbol{x}}\left(\boldsymbol{u}^{(0)}(\varepsilon \boldsymbol{y})\right)=\sum_{i=0}^{K} \varepsilon^{i} \boldsymbol{\omega}_{0}^{(i)}(\boldsymbol{y})+O\left(\varepsilon^{K+1}\right),
$$

and

$$
-\operatorname{div}_{\boldsymbol{x}}\left(\boldsymbol{E}_{f}: \boldsymbol{\epsilon}\left(\boldsymbol{u}^{(0)}\right)\right)(\varepsilon \boldsymbol{y})=\sum_{i=0}^{K} \varepsilon^{i} \boldsymbol{\gamma}^{(i)}(\boldsymbol{y})+O\left(\varepsilon^{K+1}\right) .
$$




\subsubsection{First-order approximation}

The first-order approximation is constructed by compensating the first term in the expansion of $\left[\left(\boldsymbol{E}_{m}-\boldsymbol{E}_{f}\right): \boldsymbol{\epsilon}\left(\boldsymbol{u}^{(0)}\right)(\varepsilon \boldsymbol{y})\right] \cdot \boldsymbol{n}_{f}$ and the first one in the expansion of $-\operatorname{div}_{\boldsymbol{x}}\left(\boldsymbol{E}_{f}: \boldsymbol{\epsilon}\left(\boldsymbol{u}^{(0)}\right)\right)(\varepsilon \boldsymbol{y})$. With $\boldsymbol{V}^{(0)}$ solving Problem (4.5) for $\boldsymbol{\varphi}^{(0)}(\boldsymbol{y})=\left[\left(\boldsymbol{E}_{m}-\boldsymbol{E}_{f}\right): \boldsymbol{\omega}_{0}^{(0)}(y)\right] \cdot \boldsymbol{n}_{f}$ and $\boldsymbol{Z}^{(0)}$ solving Problem (4.6) for $\boldsymbol{\kappa}^{(0)}(\boldsymbol{y})=\boldsymbol{\gamma}^{(0)}(\boldsymbol{y})$ we update the residual:

$$
\boldsymbol{d}_{1, k}^{\varepsilon}(\boldsymbol{x})=\boldsymbol{u}_{k}^{\varepsilon}(\boldsymbol{x})-\boldsymbol{u}^{(0)}(\boldsymbol{x})-\varepsilon \boldsymbol{V}_{k}^{(0)}\left(\frac{\boldsymbol{x}}{\varepsilon}\right)-\varepsilon^{2} \boldsymbol{Z}_{k}^{(0)}\left(\frac{\boldsymbol{x}}{\varepsilon}\right), \quad k \in\{f, m\} .
$$

The update of the difference allows us to obtain the first-order approximation:

$$
\boldsymbol{u}_{\varepsilon, k}^{[1]}(\boldsymbol{x})=\boldsymbol{u}^{(0)}(\boldsymbol{x})+\varepsilon \boldsymbol{V}_{k}^{(0)}\left(\frac{\boldsymbol{x}}{\varepsilon}\right)+\varepsilon^{2} \boldsymbol{Z}_{k}^{(0)}\left(\frac{\boldsymbol{x}}{\varepsilon}\right), \quad k \in\{f, m\} .
$$

Then, for $k \in\{f, m\}, \boldsymbol{d}_{1, k}^{\varepsilon}$ is solution to:

$$
\begin{aligned}
& \operatorname{div}_{\boldsymbol{x}} \boldsymbol{\sigma}_{f}\left(\boldsymbol{d}_{1, f}^{\varepsilon}\right)(\boldsymbol{x})=-\operatorname{div}_{\boldsymbol{x}}\left(\boldsymbol{E}_{f}: \boldsymbol{\epsilon}\left(\boldsymbol{u}^{(0)}\right)\right)(\varepsilon \boldsymbol{y})-\gamma_{0}^{(0)}(\boldsymbol{y}) \quad \text { in } \Omega_{f}^{\varepsilon}, \\
& \operatorname{div}{ }_{\boldsymbol{x}} \boldsymbol{\sigma}_{m}\left(\boldsymbol{d}_{1, m}^{\varepsilon}\right)(\boldsymbol{x})=\mathbf{0} \quad \text { in } \Omega_{m}^{\varepsilon}, \\
& \boldsymbol{d}_{1, f}^{\varepsilon}(\boldsymbol{x})=\boldsymbol{d}_{1, m}^{\varepsilon}(\boldsymbol{x}) \quad \text { on } \Gamma^{\varepsilon}, \\
& \boldsymbol{\sigma}_{f}\left(\boldsymbol{d}_{1, f}^{\varepsilon}\right)(\boldsymbol{x}) \cdot \boldsymbol{n}_{f}=-\boldsymbol{\sigma}_{m}\left(\boldsymbol{d}_{1, m}^{\varepsilon}\right)(\boldsymbol{x}) \cdot \boldsymbol{n}_{m} \\
& +\left(\boldsymbol{E}_{m}-\boldsymbol{E}_{\boldsymbol{f}}\right):\left[\boldsymbol{\epsilon}_{x}\left(\boldsymbol{u}^{(0)}(\varepsilon \boldsymbol{y})\right)-\boldsymbol{\omega}_{0}^{(0)}(\boldsymbol{y})\right] \cdot \boldsymbol{n}_{f} \quad \text { on } \Gamma^{\varepsilon}, \\
& \boldsymbol{d}_{1, m}^{\varepsilon}(\boldsymbol{x})=-\varepsilon \boldsymbol{V}_{m}^{(0)}\left(\frac{\boldsymbol{x}}{\varepsilon}\right)-\varepsilon^{2} \boldsymbol{Z}_{m}^{(0)}\left(\frac{\boldsymbol{x}}{\varepsilon}\right) \quad \text { on } \Gamma \text {. }
\end{aligned}
$$

\subsubsection{Second-order approximation}

We have built a first-order approximation of the solution with $\boldsymbol{u}^{(0)}, \boldsymbol{V}^{(0)}$ and $\boldsymbol{Z}^{(0)}$ independent of $\varepsilon$. The next step is to build the second-order approximation by compensating $\left(\boldsymbol{E}_{m}-\boldsymbol{E}_{\boldsymbol{f}}\right)$ :

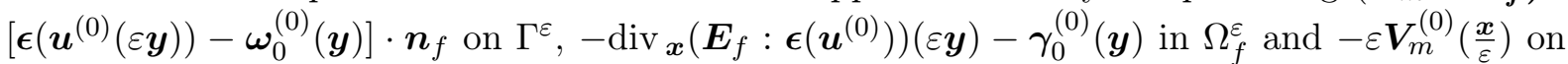
$\Gamma$. For that we introduce equivalent functions that play the same role as those introduced before but this time on $\Gamma$ :

$$
\begin{aligned}
\operatorname{div}{ }_{\boldsymbol{x}} \boldsymbol{\sigma}_{m}\left(\boldsymbol{v}^{(q)}\right)(\boldsymbol{x})=\mathbf{0} & \text { in } \Omega, \\
\boldsymbol{v}^{(q)}(\boldsymbol{x})=\boldsymbol{\psi}^{(q)}(\boldsymbol{x}) & \text { on } \Gamma .
\end{aligned}
$$

We need to expand $\boldsymbol{V}_{m}^{(0)}$ at infinity:

$$
\boldsymbol{V}_{m}^{(0)}(\boldsymbol{y})=\sum_{i=1}^{K} \frac{\boldsymbol{a}_{0}^{(i)}(\theta)}{|\boldsymbol{y}|^{i}}+O\left(\frac{1}{|\boldsymbol{y}|^{K+1}}\right)=\sum_{i=1}^{K} \varepsilon^{i} \frac{\boldsymbol{a}_{0}^{(i)}(\theta)}{|\boldsymbol{x}|^{i}}+O\left(\varepsilon^{K+1}\right), \quad \boldsymbol{x} \in \Gamma .
$$

The previous expansion is proven in Section 4.3 in the isotropic case.

So, with $\boldsymbol{V}^{(1)}$ solution of Problem (4.5) for $\boldsymbol{\varphi}^{(1)}(\boldsymbol{y})=\left[\left(\boldsymbol{E}_{m}-\boldsymbol{E}_{f}\right): \boldsymbol{\omega}_{0}^{(1)}(y)\right] \cdot \boldsymbol{n}_{f}, \boldsymbol{Z}^{(1)}$ solution Problem (4.6) for $\boldsymbol{\kappa}^{(1)}(\boldsymbol{y})=\boldsymbol{\gamma}^{(1)}(\boldsymbol{y})$ and $\boldsymbol{v}^{(2)}$ solution of Problem (4.12) for $\boldsymbol{\psi}^{(2)}(\boldsymbol{x})=-\frac{\boldsymbol{a}_{0}^{(1)}(\theta)}{|\boldsymbol{x}|}$ we update our residual as follows:

$$
\boldsymbol{d}_{2, k}^{\varepsilon}(\boldsymbol{x})=\boldsymbol{d}_{1, k}^{\varepsilon}(\boldsymbol{x})-\varepsilon^{2} \boldsymbol{V}_{k}^{(1)}\left(\frac{\boldsymbol{x}}{\varepsilon}\right)-\varepsilon^{2} \boldsymbol{v}^{(2)}(\boldsymbol{x})-\varepsilon^{3} \boldsymbol{Z}_{k}^{(1)}\left(\frac{\boldsymbol{x}}{\varepsilon}\right) .
$$


The update of the difference allows us to obtain the second-order approximation:

$$
\boldsymbol{u}_{\varepsilon, k}^{[2]}(\boldsymbol{x})=\boldsymbol{u}^{(0)}(\boldsymbol{x})+\varepsilon \boldsymbol{V}_{k}^{(0)}\left(\frac{\boldsymbol{x}}{\varepsilon}\right)+\varepsilon^{2} \boldsymbol{Z}_{k}^{(0)}\left(\frac{\boldsymbol{x}}{\varepsilon}\right)+\varepsilon^{2} \boldsymbol{V}_{k}^{(1)}\left(\frac{\boldsymbol{x}}{\varepsilon}\right)+\varepsilon^{2} \boldsymbol{v}^{(2)}(\boldsymbol{x})+\varepsilon^{3} \boldsymbol{Z}_{k}^{(1)}\left(\frac{\boldsymbol{x}}{\varepsilon}\right), \quad k \in\{f, m\} .
$$

Then, for $k \in\{f, m\}, \boldsymbol{d}_{2, k}^{\varepsilon}$ solves the following problem:

$$
\begin{aligned}
& \operatorname{div}{ }_{\boldsymbol{x}} \boldsymbol{\sigma}_{f}\left(\boldsymbol{d}_{2, f}^{\varepsilon}\right)(\boldsymbol{x})=-\operatorname{div}{ }_{\boldsymbol{x}}\left(\boldsymbol{E}_{f}: \boldsymbol{\epsilon}\left(\boldsymbol{u}^{(0)}\right)\right)(\varepsilon \boldsymbol{y})-\boldsymbol{\gamma}_{0}^{(0)}(\boldsymbol{y})-\varepsilon \boldsymbol{\gamma}_{0}^{(1)}(\boldsymbol{y}) \quad \text { in } \Omega_{f}^{\varepsilon}, \\
& \operatorname{div}{ }_{\boldsymbol{x}} \boldsymbol{\sigma}_{m}\left(\boldsymbol{d}_{2, m}^{\varepsilon}\right)(\boldsymbol{x})=\mathbf{0} \quad \text { in } \Omega_{m}^{\varepsilon}, \\
& \boldsymbol{d}_{2, f}^{\varepsilon}(\boldsymbol{x})=\boldsymbol{d}_{2, m}^{\varepsilon}(\boldsymbol{x}) \quad \text { on } \Gamma^{\varepsilon}, \\
& \boldsymbol{\sigma}_{f}\left(\boldsymbol{d}_{2, f}^{\varepsilon}\right)(\boldsymbol{x}) \cdot \boldsymbol{n}_{f}=-\boldsymbol{\sigma}_{m}\left(\boldsymbol{d}_{2, m}^{\varepsilon}\right)(\boldsymbol{x}) \cdot \boldsymbol{n}_{m} \\
& \left.+\left(\boldsymbol{E}_{m}-\boldsymbol{E}_{f}\right):\left[\boldsymbol{\epsilon}\left(\boldsymbol{u}^{(0)}(\boldsymbol{\varepsilon} \boldsymbol{y})\right)\right)-\boldsymbol{\omega}_{0}^{(0)}(\boldsymbol{y})-\varepsilon \boldsymbol{\omega}_{0}^{(1)}(\boldsymbol{y})\right] \cdot \boldsymbol{n}_{f} \\
& +\varepsilon^{2}\left(\boldsymbol{E}_{m}-\boldsymbol{E}_{f}\right):\left[\boldsymbol{\epsilon}\left(\boldsymbol{v}^{(2)}(\varepsilon \boldsymbol{y})\right)\right] \cdot \boldsymbol{n}_{f} \quad \text { on } \Gamma^{\varepsilon}, \\
& \boldsymbol{d}_{2, m}^{\varepsilon}(\boldsymbol{x})=\varepsilon\left(\varepsilon \frac{\boldsymbol{a}_{0}^{(1)}(\theta)}{|\boldsymbol{x}|}-\boldsymbol{V}_{m}^{\left.(0)\left(\frac{\boldsymbol{x}}{\varepsilon}\right)\right)}\right. \\
& -\varepsilon^{2}\left(\boldsymbol{Z}_{m}^{(0)}\left(\frac{\boldsymbol{x}}{\varepsilon}\right)+\boldsymbol{V}_{m}^{(1)}\left(\frac{\boldsymbol{x}}{\varepsilon}\right)\right)-\varepsilon^{3} \boldsymbol{Z}_{m}^{(1)}\left(\frac{\boldsymbol{x}}{\varepsilon}\right) \quad \text { on } \Gamma \text {. }
\end{aligned}
$$

\subsubsection{Third-order approximation}

Following the same approach, we expand as done before $\boldsymbol{\epsilon}\left(\boldsymbol{v}^{(2)}\right)(\varepsilon \boldsymbol{y})$ as follows:

$$
\boldsymbol{\epsilon}\left(\boldsymbol{v}^{(2)}\right)(\varepsilon \boldsymbol{y})=\sum_{i=0}^{K} \varepsilon^{(i)} \boldsymbol{\omega}_{2}^{(i)}(y)+O\left(\varepsilon^{K+1}\right)
$$

Now, we need to expand $\boldsymbol{V}_{m}^{(1)}+\boldsymbol{Z}_{m}^{(0)}$ at infinity since both functions have a factor of $\varepsilon^{2}$ :

$$
\boldsymbol{V}_{m}^{(1)}(\boldsymbol{y})+\boldsymbol{Z}_{m}^{(0)}(\boldsymbol{y})=\sum_{i=1}^{K} \frac{\boldsymbol{a}_{1}^{(i)}(\theta)}{|\boldsymbol{y}|^{(i)}}+O\left(\frac{1}{|\boldsymbol{y}|^{K+1}}\right)=\sum_{i=1}^{K} \varepsilon^{i} \frac{\boldsymbol{a}_{1}^{(i)}(\theta)}{|\boldsymbol{x}|^{i}}+O\left(\varepsilon^{K+1}\right), \quad \boldsymbol{x} \in \Gamma .
$$

The previous expansion is proven in Section 4.3 in the isotropic case.

With $\boldsymbol{V}^{(2)}$ solution of Problem (4.5) for $\boldsymbol{\varphi}^{(2)}(\boldsymbol{y})=\left(\boldsymbol{E}_{m}-\boldsymbol{E}_{f}\right):\left(\boldsymbol{\omega}_{0}^{(2)}(\boldsymbol{y})+\boldsymbol{\omega}_{2}^{(0)}(\boldsymbol{y})\right) \cdot \boldsymbol{n}_{f}, \boldsymbol{Z}^{(2)}$ solving Problem (4.6) for $\boldsymbol{\kappa}^{(2)}(\boldsymbol{y})=\gamma^{(2)}(\boldsymbol{y})$ and $\boldsymbol{v}^{(3)}$ solution of Problem (4.12) for $\boldsymbol{\psi}^{(3)}=$ $-\frac{\boldsymbol{a}_{1}^{(1)}(\theta)}{|\boldsymbol{x}|}-\frac{\boldsymbol{a}_{0}^{(2)}(\theta)}{|\boldsymbol{x}|^{2}}$ we write the third-order update:

$$
\boldsymbol{d}_{3, k}^{\varepsilon}(\boldsymbol{x})=\boldsymbol{d}_{2, k}^{\varepsilon}(\boldsymbol{x})-\varepsilon^{3} \boldsymbol{v}^{(3)}(\boldsymbol{x})-\varepsilon^{3} \boldsymbol{V}_{k}^{(2)}\left(\frac{\boldsymbol{x}}{\varepsilon}\right)-\varepsilon^{4} \boldsymbol{Z}_{k}^{(2)}\left(\frac{\boldsymbol{x}}{\varepsilon}\right), \quad k \in\{f, m\} .
$$

The update of the difference allows us to obtain the third-order approximation:

$$
\begin{aligned}
\boldsymbol{u}_{\varepsilon, k}^{[3]}(\boldsymbol{x})=\boldsymbol{u}^{(0)} & (\boldsymbol{x})+\varepsilon \boldsymbol{V}_{k}^{(0)}\left(\frac{\boldsymbol{x}}{\varepsilon}\right)+\varepsilon^{2} \boldsymbol{Z}_{k}^{(0)}\left(\frac{\boldsymbol{x}}{\varepsilon}\right)+\varepsilon^{2} \boldsymbol{V}_{k}^{(1)}\left(\frac{\boldsymbol{x}}{\varepsilon}\right)+\varepsilon^{2} \boldsymbol{v}^{(2)}(\boldsymbol{x}) \\
& +\varepsilon^{3} \boldsymbol{Z}_{k}^{(1)}\left(\frac{\boldsymbol{x}}{\varepsilon}\right)+\varepsilon^{3} \boldsymbol{v}^{(3)}(\boldsymbol{x})+\varepsilon^{3} \boldsymbol{V}_{k}^{(2)}\left(\frac{\boldsymbol{x}}{\varepsilon}\right)+\varepsilon^{4} \boldsymbol{Z}_{k}^{(2)}\left(\frac{\boldsymbol{x}}{\varepsilon}\right), \quad k \in\{f, m\}
\end{aligned}
$$


Then, for $k \in\{f, m\}, \boldsymbol{d}_{3, k}^{\varepsilon}$ solves the following problem:

$$
\begin{aligned}
& \operatorname{div}{ }_{\boldsymbol{x}} \boldsymbol{\sigma}_{f}\left(\boldsymbol{d}_{3, f}^{\varepsilon}\right)(\boldsymbol{x})=-\operatorname{div}_{\boldsymbol{x}}\left(\boldsymbol{E}_{f}: \boldsymbol{\epsilon}\left(\boldsymbol{u}^{(0)}\right)\right)(\varepsilon \boldsymbol{y})-\gamma_{0}^{(0)}(\boldsymbol{y}) \\
& -\varepsilon \gamma_{0}^{(1)}(\boldsymbol{y})-\varepsilon^{2} \gamma_{0}^{(2)}(\boldsymbol{y}) \quad \text { in } \Omega_{f}^{\varepsilon}, \\
& \operatorname{div}{ }_{\boldsymbol{x}} \boldsymbol{\sigma}_{2}\left(\boldsymbol{d}_{3, m}^{\varepsilon}\right)(\boldsymbol{x})=\mathbf{0} \quad \text { in } \Omega_{m}^{\varepsilon} \text {, } \\
& \boldsymbol{d}_{3, f}^{\varepsilon}(\boldsymbol{x})=\boldsymbol{d}_{3, m}^{\varepsilon}(\boldsymbol{x}) \quad \text { on } \Gamma^{\varepsilon}, \\
& \boldsymbol{\sigma}_{f}\left(\boldsymbol{d}_{3, f}^{\varepsilon}\right)(\boldsymbol{x}) \cdot \boldsymbol{n}_{f}=-\boldsymbol{\sigma}_{m}\left(\boldsymbol{d}_{3, m}^{\varepsilon}\right)(\boldsymbol{x}) \cdot \boldsymbol{n}_{m} \\
& +\left(\boldsymbol{E}_{m}-\boldsymbol{E}_{f}\right):\left[\varepsilon\left(\boldsymbol{u}^{(0)}\right)(\varepsilon \boldsymbol{y})-\boldsymbol{\omega}_{0}^{(0)}(\boldsymbol{y})-\varepsilon \boldsymbol{\omega}_{0}^{(1)}(\boldsymbol{y})-\varepsilon^{2} \boldsymbol{\omega}_{0}^{(2)}(\boldsymbol{y})\right] \cdot \boldsymbol{n}_{f} \\
& +\varepsilon^{2}\left(\boldsymbol{E}_{m}-\boldsymbol{E}_{f}\right):\left[\boldsymbol{\epsilon}\left(\boldsymbol{v}^{(2)}(\varepsilon \boldsymbol{y})\right)-\boldsymbol{\omega}_{2}^{(0)}(\boldsymbol{y})\right] \cdot \boldsymbol{n}_{f} \\
& +\varepsilon^{3}\left(\boldsymbol{E}_{m}-\boldsymbol{E}_{f}\right):\left[\boldsymbol{\epsilon}\left(\boldsymbol{v}^{(3)}(\varepsilon \boldsymbol{y})\right)\right] \cdot \boldsymbol{n}_{f} \quad \text { on } \Gamma^{\varepsilon}, \\
& \boldsymbol{d}_{3, m}^{\varepsilon}(\boldsymbol{x})=\varepsilon\left(\varepsilon^{2} \frac{\boldsymbol{a}_{0}^{(2)}(\theta)}{|\boldsymbol{x}|^{2}}+\varepsilon \frac{\boldsymbol{a}_{0}^{(1)}(\theta)}{|\boldsymbol{x}|}-\boldsymbol{V}_{m}^{(0)}\left(\frac{\boldsymbol{x}}{\varepsilon}\right)\right) \\
& +\varepsilon^{2}\left(\varepsilon \frac{\boldsymbol{a}_{1}^{(1)}(\theta)}{|\boldsymbol{x}|}-\boldsymbol{V}_{m}^{(1)}\left(\frac{\boldsymbol{x}}{\varepsilon}\right)-\boldsymbol{Z}_{m}^{(0)}\left(\frac{\boldsymbol{x}}{\varepsilon}\right)\right) \\
& -\varepsilon^{3}\left(\boldsymbol{V}_{m}^{(2)}\left(\frac{\boldsymbol{x}}{\varepsilon}\right)+\boldsymbol{Z}_{m}^{(1)}\left(\frac{\boldsymbol{x}}{\varepsilon}\right)\right)-\varepsilon^{4} \boldsymbol{Z}_{m}^{(2)}\left(\frac{\boldsymbol{x}}{\varepsilon}\right) \quad \text { on } \Gamma \text {. }
\end{aligned}
$$

\subsubsection{Fourth-order approximation}

As done for the Poisson problem, we go there to the fourth-order to deduce the general form of the function $\boldsymbol{\psi}$. Now we expand $\boldsymbol{\epsilon}\left(\boldsymbol{v}^{(3)}\right)(\varepsilon \boldsymbol{y})$ :

$$
\boldsymbol{\epsilon}\left(\boldsymbol{v}^{(3)}\right)(\varepsilon \boldsymbol{y})=\sum_{i=0}^{K} \varepsilon^{i} \boldsymbol{\omega}_{3}^{(i)}(y)+O\left(\varepsilon^{K+1}\right)
$$

We need to expand too $\boldsymbol{V}_{m}^{(2)}+\boldsymbol{Z}_{m}^{(1)}$ at infinity since both functions have a factor of $\varepsilon^{3}$ :

$$
\boldsymbol{V}_{m}^{(2)}(\boldsymbol{y})+\boldsymbol{Z}_{m}^{(1)}(\boldsymbol{y})=\sum_{i=1}^{K} \frac{\boldsymbol{a}_{2}^{(i)}(\theta)}{|\boldsymbol{y}|^{i}}+O\left(\frac{1}{|\boldsymbol{y}|^{K+1}}\right)=\sum_{i=1}^{K} \varepsilon^{i} \frac{\boldsymbol{a}_{2}^{(i)}(\theta)}{|\boldsymbol{x}|^{i}}+O\left(\varepsilon^{K+1}\right), \quad \boldsymbol{x} \in \Gamma .
$$

The previous expansion is proven in Section 4.3 in the isotropic case.

Finally, with $\boldsymbol{V}^{(3)}$ solution of Problem (4.5) for $\boldsymbol{\varphi}^{(3)}(\boldsymbol{y})=\left(\boldsymbol{E}_{m}-\boldsymbol{E}_{f}\right):\left(\boldsymbol{\omega}_{0}^{(3)}(\boldsymbol{y})+\boldsymbol{\omega}_{2}^{(1)}(\boldsymbol{y})+\right.$ $\left.\boldsymbol{\omega}_{3}^{(0)}(\boldsymbol{y})\right) \cdot \boldsymbol{n}_{f}, \boldsymbol{Z}^{(3)}$ solving Problem (4.6) for $\boldsymbol{\kappa}^{(3)}(\boldsymbol{y})=\gamma_{0}^{(3)}(\boldsymbol{y})$ and $\boldsymbol{v}^{(3)}$ solution of Problem (4.12) for $\boldsymbol{\psi}^{(3)}=-\frac{\boldsymbol{a}_{2}^{(1)}(\theta)}{|\boldsymbol{x}|}-\frac{\boldsymbol{a}_{1}^{(2)}(\theta)}{|\boldsymbol{x}|^{2}}-\frac{\boldsymbol{a}_{0}^{(3)}(\theta)}{|\boldsymbol{x}|^{3}}$ we write the fourth-order update:

$$
\boldsymbol{d}_{4, k}^{\varepsilon}(\boldsymbol{x})=\boldsymbol{d}_{3, k}^{\varepsilon}(\boldsymbol{x})-\varepsilon^{4} \boldsymbol{v}^{(4)}(\boldsymbol{x})-\varepsilon^{4} \boldsymbol{V}_{k}^{(3)}\left(\frac{\boldsymbol{x}}{\varepsilon}\right)-\varepsilon^{5} \boldsymbol{Z}_{k}^{(3)}\left(\frac{\boldsymbol{x}}{\varepsilon}\right), \quad k \in\{f, m\} .
$$


We obtain the following equations for $\boldsymbol{d}_{4, k}^{\varepsilon}(\boldsymbol{x}), k \in\{f, m\}$ :

$$
\begin{aligned}
& \operatorname{div} \boldsymbol{\sigma}_{f}\left(\boldsymbol{d}_{4, f}^{\varepsilon}\right)(\boldsymbol{x})=-\operatorname{div}_{\boldsymbol{x}}\left(\boldsymbol{E}_{f}: \boldsymbol{\epsilon}\left(\boldsymbol{u}^{(0)}\right)\right)(\varepsilon \boldsymbol{y})-\gamma_{0}^{(0)}(\boldsymbol{y})-\varepsilon \boldsymbol{\gamma}_{0}^{(1)}(\boldsymbol{y}) \\
& -\varepsilon^{2} \gamma_{0}^{(2)}(\boldsymbol{y})-\varepsilon^{3} \boldsymbol{\gamma}_{0}^{(3)}(\boldsymbol{y}) \quad \text { in } \Omega_{f}^{\varepsilon},(4.25 \mathrm{a}) \\
& \operatorname{div} \boldsymbol{\sigma}_{m}\left(\boldsymbol{d}_{4, m}^{\varepsilon}\right)(\boldsymbol{x})=\mathbf{0} \quad \text { in } \Omega_{m}^{\varepsilon},(4.25 \mathrm{~b}) \\
& \boldsymbol{d}_{4, f}^{\varepsilon}(\boldsymbol{x})=\boldsymbol{d}_{4, m}^{\varepsilon}(\boldsymbol{x}) \quad \text { on } \Gamma^{\varepsilon},(4.25 \mathrm{c}) \\
& \boldsymbol{\sigma}_{f}\left(\boldsymbol{d}_{4, f}^{\varepsilon}\right)(\boldsymbol{x}) \cdot \boldsymbol{n}_{f}=-\boldsymbol{\sigma}_{m}\left(\boldsymbol{d}_{4, m}^{\varepsilon}\right)(\boldsymbol{x}) \cdot \boldsymbol{n}_{m} \\
& +\left(\boldsymbol{E}_{m}-\boldsymbol{E}_{f}\right):\left[\left(\boldsymbol{\epsilon}\left(\boldsymbol{u}^{(0)}(\varepsilon \boldsymbol{y})\right)-\boldsymbol{\omega}_{0}^{(0)}(\boldsymbol{y})-\varepsilon \boldsymbol{\omega}_{0}^{(1)}(\boldsymbol{y})-\varepsilon^{2} \boldsymbol{\omega}_{0}^{(2)}(\boldsymbol{y})-\varepsilon^{3} \boldsymbol{\omega}_{0}^{(3)}(\boldsymbol{y})\right] \cdot \boldsymbol{n}_{f}\right. \\
& \left.+\varepsilon^{2}\left(\boldsymbol{E}_{m}-\boldsymbol{E}_{f}\right):\left[\left(\boldsymbol{\epsilon}\left(\boldsymbol{v}^{(2)}(\varepsilon \boldsymbol{y})\right)-\boldsymbol{\omega}_{2}^{(0)}(\boldsymbol{y})\right)-\varepsilon \boldsymbol{\omega}_{2}^{(1)}(\boldsymbol{y})\right)\right] \cdot \boldsymbol{n}_{f} \\
& +\varepsilon^{3}\left(\boldsymbol{E}_{m}-\boldsymbol{E}_{f}\right):\left[\boldsymbol{\epsilon}\left(\boldsymbol{v}^{(3)}(\varepsilon \boldsymbol{y})\right)-\boldsymbol{\omega}_{3}^{(0)}(\boldsymbol{y})\right] \cdot \boldsymbol{n}_{f} \\
& +\varepsilon^{4}\left(\boldsymbol{E}_{m}-\boldsymbol{E}_{f}\right):\left[\boldsymbol{\epsilon}\left(\boldsymbol{v}^{(4)}(\varepsilon \boldsymbol{y})\right)\right] \cdot \boldsymbol{n}_{f} \quad \text { on } \Gamma^{\varepsilon},(4.25 \mathrm{~d}) \\
& \boldsymbol{d}_{4, m}^{\varepsilon}(\boldsymbol{x})=\varepsilon\left(\varepsilon^{3} \frac{\boldsymbol{a}_{0}^{(3)}(\theta)}{|\boldsymbol{x}|^{3}}+\varepsilon^{2} \frac{\boldsymbol{a}_{0}^{(2)}(\theta)}{|\boldsymbol{x}|^{2}}+\varepsilon \frac{\boldsymbol{a}_{0}^{(1)}(\theta)}{|\boldsymbol{x}|}-\boldsymbol{V}_{m}^{(0)}\left(\frac{\boldsymbol{x}}{\varepsilon}\right)\right) \\
& +\varepsilon^{2}\left(\varepsilon^{2} \frac{\boldsymbol{a}_{1}^{(2)}(\theta)}{|\boldsymbol{x}|^{2}}+\varepsilon \frac{\boldsymbol{a}_{1}^{(1)}(\theta)}{|\boldsymbol{x}|}-\boldsymbol{V}_{m}^{(1)}\left(\frac{\boldsymbol{x}}{\varepsilon}\right)-\boldsymbol{Z}_{m}^{(0)}\left(\frac{\boldsymbol{x}}{\varepsilon}\right)\right) \\
& +\varepsilon^{3}\left(\varepsilon \frac{\boldsymbol{a}_{2}^{(1)}(\theta)}{|\boldsymbol{x}|}-\boldsymbol{V}_{m}^{(2)}\left(\frac{\boldsymbol{x}}{\varepsilon}\right)-\boldsymbol{Z}_{m}^{(1)}\left(\frac{\boldsymbol{x}}{\varepsilon}\right)\right) \\
& -\varepsilon^{3}\left(\boldsymbol{V}_{m}^{(3)}\left(\frac{\boldsymbol{x}}{\varepsilon}\right)+\boldsymbol{Z}_{m}^{(2)}\left(\frac{\boldsymbol{x}}{\varepsilon}\right)\right)-\varepsilon^{5} \boldsymbol{Z}_{m}^{(3)}\left(\frac{\boldsymbol{x}}{\varepsilon}\right) \quad \text { on } \Gamma \text {. }
\end{aligned}
$$

\subsubsection{The $K$-th order approximation}

We can update our residual at the order $n \geq 3$ as follows:

$$
\boldsymbol{d}_{n, k}^{\varepsilon}(\boldsymbol{x})=\boldsymbol{d}_{n-1, k}^{\varepsilon}(\boldsymbol{x})-\varepsilon^{n} \boldsymbol{v}_{k}^{(n)}(\boldsymbol{x})-\varepsilon^{n} \boldsymbol{V}_{k}^{(n-1)}\left(\frac{\boldsymbol{x}}{\varepsilon}\right)-\varepsilon^{n+1} \boldsymbol{Z}_{k}^{(n-1)}\left(\frac{\boldsymbol{x}}{\varepsilon}\right), \quad k \in\{f, m\} .
$$

With $\boldsymbol{v}^{(n)}$ solves Problem (4.12) for $\boldsymbol{\psi}^{(n)}=-\sum_{\substack{p+q=n-1 \\ p \geq 1, q \geq 0}} \frac{\boldsymbol{a}_{q}^{(p)}(\theta)}{|\boldsymbol{x}|^{p}}, \boldsymbol{V}^{(n-1)}$ the solution of Problem (4.5) with $\boldsymbol{\varphi}^{(n-1)}(\boldsymbol{y})=\left(\boldsymbol{E}_{m}-\boldsymbol{E}_{\boldsymbol{f}}\right): \sum_{\substack{p+q=n-1 \\ p \geq 1, q \geq 0}} \boldsymbol{\omega}_{q}^{(p)}(\boldsymbol{y}) \cdot \boldsymbol{n}_{f}$, and $\boldsymbol{Z}^{(n-1)}$ solving Problem (4.6) for $\boldsymbol{\kappa}^{(n-1)}(\boldsymbol{y})=\boldsymbol{\gamma}^{(n-1)}(\boldsymbol{y})$. We are now able to build an approximation $\boldsymbol{u}_{\varepsilon}^{[K]}(\boldsymbol{x})$ to the order $K \geq 3(k \in\{f, m\})$ :

$$
\boldsymbol{u}_{\varepsilon, k}^{[K]}(\boldsymbol{x})=\boldsymbol{u}^{(0)}(\boldsymbol{x})+\sum_{i=2}^{K} \varepsilon^{i} \boldsymbol{v}^{(i)}(\boldsymbol{x})+\sum_{i=1}^{K} \varepsilon^{i} \boldsymbol{V}_{k}^{(i-1)}\left(\frac{\boldsymbol{x}}{\varepsilon}\right)+\sum_{i=1}^{K} \varepsilon^{i+1} \boldsymbol{Z}_{k}^{(i-1)}\left(\frac{\boldsymbol{x}}{\varepsilon}\right) .
$$

We obtain the following equations for $\boldsymbol{d}_{K, k}^{\varepsilon}(k \in\{f, m\})$ :

$$
\begin{array}{rr}
\operatorname{div} \boldsymbol{\sigma}_{f}\left(\boldsymbol{d}_{K, f}^{\varepsilon}\right)(\boldsymbol{x})=O_{H^{-1}\left(\Omega ; \mathbb{R}^{2}\right)}\left(\varepsilon^{K+1}\right) & \text { in } \Omega_{f}^{\varepsilon}, \\
\operatorname{div} \boldsymbol{\sigma}_{m}\left(\boldsymbol{d}_{K, m}^{\varepsilon}\right)(\boldsymbol{x})=\mathbf{0} & \text { in } \Omega_{m}^{\varepsilon}, \\
\boldsymbol{d}_{K, f}^{\varepsilon}(\boldsymbol{x})=\boldsymbol{d}_{K, m}^{\varepsilon}(\boldsymbol{x}) & \text { on } \Gamma^{\varepsilon}, \\
\boldsymbol{\sigma}_{f}\left(\boldsymbol{d}_{K, f}^{\varepsilon}\right)(\boldsymbol{x}) \cdot \boldsymbol{n}_{f}=-\boldsymbol{\sigma}_{m}\left(\boldsymbol{d}_{K, m}^{\varepsilon}\right)(\boldsymbol{x}) \cdot \boldsymbol{n}_{m}+O_{H^{-\frac{1}{2}}\left(\Gamma, \mathbb{R}^{2}\right)}\left(\varepsilon^{K+1}\right), & \\
\boldsymbol{d}_{K, m}^{\varepsilon}(\boldsymbol{x})=O_{H^{\frac{1}{2}}\left(\Gamma, \mathbb{R}^{2}\right)}\left(\varepsilon^{K+1}\right) & \text { on } \Gamma .
\end{array}
$$




\subsection{Existence and uniqueness in unbounded domains for the linear elasticity problem}

A weak formulation of Problem (4.5) writes:

$$
\int_{\Omega_{f}^{1}} \boldsymbol{\epsilon}\left(\boldsymbol{V}_{f}^{(p)}\right): \boldsymbol{E}_{f}: \boldsymbol{\epsilon}(\boldsymbol{\omega}) \mathrm{d} \boldsymbol{x}+\int_{\Omega^{\infty}} \boldsymbol{\epsilon}\left(\boldsymbol{V}_{m}^{(p)}\right): \boldsymbol{E}_{m}: \boldsymbol{\epsilon}(\boldsymbol{\omega}) \mathrm{d} \boldsymbol{x}=\int_{\Gamma^{1}} \boldsymbol{\varphi} \cdot \boldsymbol{w} \mathrm{d} \sigma, \quad \text { for all } \boldsymbol{w} \in V
$$

Introducing the space $\mathbb{V}=\left\{\boldsymbol{v} \in\left(\mathcal{W}_{0,0}^{1,2}\right)^{2} ; \int_{\Gamma_{R_{0}}} \boldsymbol{v} \mathrm{d} \sigma=\mathbf{0}\right\}$ we can see that the bilinear form $\int_{\Omega_{f}^{1}} \boldsymbol{\epsilon}\left(\boldsymbol{V}_{f}^{(p)}\right): \boldsymbol{E}_{f}: \boldsymbol{\epsilon}(\boldsymbol{\omega}) \mathrm{d} \boldsymbol{x}+\int_{\Omega^{\infty}} \boldsymbol{\epsilon}\left(\boldsymbol{V}_{m}^{(p)}\right): \boldsymbol{E}_{m}: \boldsymbol{\epsilon}(\boldsymbol{\omega}) \mathrm{d} \boldsymbol{x}$ is coercive on $\mathbb{V}$. In addition, this bilinear form and the linear form $\int_{\Gamma^{1}} \boldsymbol{\varphi} \cdot \boldsymbol{w} \mathrm{d} \sigma$ are continuous on $\mathbb{V}$. Then, the existence and uniqueness of the function $\boldsymbol{V}^{(p)}$ in $\mathbb{V}$ solution to (4.29) follow from the Lax-Milgram theorem. The existence and uniqueness of $\boldsymbol{Z}^{(p)}$ are proven in the same way.

\subsection{Expansion of the functions $\boldsymbol{V}_{m}^{(p)}$ and $\boldsymbol{Z}_{m}^{(p)}$ at infinity}

We restrict here to the isotropic case where $\boldsymbol{E}_{m}: \boldsymbol{\epsilon}=\lambda \operatorname{trace}(\boldsymbol{\epsilon}) I+2 \mu \boldsymbol{\epsilon}$, for $\lambda, \mu$ the Lamé coefficients. In that case, according to [30] the functions

$$
\begin{aligned}
& \psi_{1}^{(s)}(\theta)=\left(\begin{array}{c}
\sin ((s+1) \theta) \\
\cos ((s+1) \theta)
\end{array}\right), \\
& \psi_{2}^{(s)}(\theta)=\left(\begin{array}{c}
\cos ((s+1) \theta) \\
-\sin ((s+1) \theta)
\end{array}\right), \\
& \psi_{3}^{(s)}(\theta)=\left(\begin{array}{c}
(1-s+2 \rho) \sin ((s-1) \theta) \\
-(1+s+2 \rho) \cos ((s-1) \theta)
\end{array}\right), \\
& \psi_{4}^{(s)}(\theta)=\left(\begin{array}{c}
-(1-s+2 \rho) \cos ((s-1) \theta) \\
-(1+s+2 \rho) \sin ((s-1) \theta)
\end{array}\right),
\end{aligned}
$$

whith $\rho=\frac{\mu}{(\lambda+\mu)}$, generate the expansion at infinity of the functions $\boldsymbol{V}_{m}^{(p)}$ and $\boldsymbol{Z}_{m}^{(p)}$ which takes the following form:

$$
\boldsymbol{V}_{m}^{(p)}=\boldsymbol{Z}_{m}^{(p)}=\sum_{s \in \mathbb{N}^{*}} r^{-s}\left(a_{s} \boldsymbol{\psi}_{1}^{(-s)}+b_{s} \boldsymbol{\psi}_{2}^{(-s)}+c_{s} \boldsymbol{\psi}_{3}^{(-s)}+d_{s} \boldsymbol{\psi}_{4}^{(-s)}\right)
$$

\subsection{Error estimate for the linear elasticity problem}

In this section we establish an error estimate for the studied problem by increasing the $H^{1}\left(\Omega ; \mathbb{R}^{2}\right)$ norm by powers of $\varepsilon$ (characteristic length of the inclusion). First, we begin by considering the following problem for a given $\boldsymbol{g} \in H^{-\frac{1}{2}}\left(\Gamma^{\varepsilon} ; \mathbb{R}^{2}\right), \boldsymbol{h} \in H^{\frac{1}{2}}\left(\Gamma ; \mathbb{R}^{2}\right)$ and $\boldsymbol{f} \in H^{-1}\left(\Omega_{f}^{\varepsilon} ; \mathbb{R}^{2}\right)$ :

$$
\begin{aligned}
\operatorname{div} \boldsymbol{\sigma}_{f}\left(\boldsymbol{z}_{f}\right)(\boldsymbol{x})=-\boldsymbol{f}(\boldsymbol{x}) & \text { in } \Omega_{f}^{\varepsilon}, \\
\operatorname{div} \boldsymbol{\sigma}_{m}\left(\boldsymbol{z}_{m}\right)(\boldsymbol{x})=\mathbf{0} & \text { in } \Omega_{m}^{\varepsilon}, \\
\boldsymbol{z}_{f}(\boldsymbol{x})=\boldsymbol{z}_{m}(\boldsymbol{x}) & \text { on } \Gamma^{\varepsilon}, \\
\boldsymbol{\sigma}_{f}\left(\boldsymbol{z}_{f}\right)(\boldsymbol{x}) \cdot \boldsymbol{n}_{f}=-\boldsymbol{\sigma}_{m}\left(\boldsymbol{z}_{m}\right)(\boldsymbol{x}) \cdot \boldsymbol{n}_{m}+\boldsymbol{g}(\boldsymbol{x}) & \text { on } \Gamma^{\varepsilon}, \\
\boldsymbol{z}_{m}(\boldsymbol{x})=\boldsymbol{h}(\boldsymbol{x}) & \text { on } \Gamma .
\end{aligned}
$$


Its weak form can be written as: Find $\boldsymbol{z} \in H^{1}\left(\Omega ; \mathbb{R}^{2}\right)$ with $\boldsymbol{z}=\boldsymbol{h}$ on $\Gamma$ such that:

$$
\int_{\Omega_{f}^{\varepsilon}} \boldsymbol{\epsilon}(\boldsymbol{z}): \boldsymbol{E}_{f}: \boldsymbol{\epsilon}(\boldsymbol{\omega}) \mathrm{d} \boldsymbol{x}+\int_{\Omega_{m}^{\varepsilon}} \boldsymbol{\epsilon}(\boldsymbol{z}): \boldsymbol{E}_{m}: \boldsymbol{\epsilon}(\boldsymbol{\omega}) \mathrm{d} \boldsymbol{x}+\int_{\Gamma^{\varepsilon}} \boldsymbol{g} \cdot \boldsymbol{\omega} \mathrm{d} \sigma=\int_{\Omega_{f}^{\varepsilon}} \boldsymbol{f} \cdot \boldsymbol{\omega} \mathrm{d} \boldsymbol{x}
$$

for all $\boldsymbol{\omega} \in H^{1}\left(\Omega ; \mathbb{R}^{2}\right)$ such that $\boldsymbol{\omega}=\mathbf{0}$ on $\Gamma$.

Lemma 4.1. There exists a unique solution $\boldsymbol{z}$ to the weak form (4.35) of Problem (4.34) satisfying

$$
\|\boldsymbol{z}\|_{H^{1}(\Omega)} \leq C\left(\|\boldsymbol{h}\|_{H^{\frac{1}{2}\left(\Gamma ; \mathbb{R}^{2}\right)}}+\|\boldsymbol{g}\|_{H^{-\frac{1}{2}}\left(\Gamma^{\varepsilon} ; \mathbb{R}^{2}\right)}+\|\boldsymbol{f}\|_{H^{-1}\left(\Omega_{f}^{\varepsilon} ; \mathbb{R}^{2}\right)}\right),
$$

where $C>0$ is a constant independent of $\varepsilon$.

Proof. The surjectivity of the trace operator guarantees the existence of a continuous lifting operator $\boldsymbol{H} \in H^{1}\left(\Omega ; \mathbb{R}^{2}\right)$ of $\boldsymbol{h}$ (see [29]). Then, $\overline{\boldsymbol{z}}=\boldsymbol{z}-\boldsymbol{H}$ solves the following equation for all $\boldsymbol{\omega} \in H^{1}\left(\Omega ; \mathbb{R}^{2}\right)$ such that $\boldsymbol{\omega}=\mathbf{0}$ on $\Gamma$ :

$$
\begin{aligned}
\int_{\Omega_{f}^{\varepsilon}} \boldsymbol{\epsilon}(\overline{\boldsymbol{z}}): \boldsymbol{E}_{f}: \boldsymbol{\epsilon}(\boldsymbol{\omega}) \mathrm{d} \boldsymbol{x} & +\int_{\Omega_{m}^{\varepsilon}} \boldsymbol{\epsilon}(\overline{\boldsymbol{z}}): \boldsymbol{E}_{m}: \boldsymbol{\epsilon}(\boldsymbol{\omega}) \mathrm{d} \boldsymbol{x}+\int_{\Omega_{f}^{\varepsilon}} \boldsymbol{\epsilon}(\overline{\boldsymbol{z}}): \boldsymbol{E}_{f}: \boldsymbol{\epsilon}(\boldsymbol{\omega}) \mathrm{d} \boldsymbol{x} \\
& +\int_{\Omega_{m}^{\varepsilon}} \boldsymbol{\epsilon}(\overline{\boldsymbol{z}}): \boldsymbol{E}_{m}: \boldsymbol{\epsilon}(\boldsymbol{\omega}) \mathrm{d} \boldsymbol{x}+\int_{\Gamma^{\varepsilon}} \boldsymbol{g} \cdot \boldsymbol{\omega} \mathrm{d} \sigma=\int_{\Omega_{f}^{\varepsilon}} \boldsymbol{f} \cdot \boldsymbol{\omega} \mathrm{d} \boldsymbol{x} .
\end{aligned}
$$

The bilinear form $a(\boldsymbol{u}, \boldsymbol{v})=\int_{\Omega_{f}^{\varepsilon}} \boldsymbol{\epsilon}(\boldsymbol{u}): \boldsymbol{E}_{f}: \boldsymbol{\epsilon}(\boldsymbol{v}) \mathrm{d} \boldsymbol{x}+\int_{\Omega_{m}^{\varepsilon}} \boldsymbol{\epsilon}(\boldsymbol{u}): \boldsymbol{E}_{m}: \boldsymbol{\epsilon}(\boldsymbol{v}) \mathrm{d} \boldsymbol{x}$ associated to the weak formulation (4.36) is coercive, i.e. $\alpha\|\boldsymbol{v}\|_{H^{1}\left(\Omega ; \mathbb{R}^{2}\right)}^{2} \leq a(\boldsymbol{v}, \boldsymbol{v})$, see e.g. [28,29]. Then, the Lax-Milgram theorem guarantees the existence and uniqueness of $\bar{z}$ solution of (4.36) and hence existence and uniqueness of solution to (4.36) follow. Using the coercivity of the bilinear form we obtain:

$$
\alpha\|\overline{\boldsymbol{z}}\|_{H^{1}\left(\Omega ; \mathbb{R}^{2}\right)}^{2} \leq-\int_{\Gamma^{\varepsilon}} \boldsymbol{g} \cdot \overline{\boldsymbol{z}} \mathrm{d} \sigma-\int_{\Omega} \boldsymbol{\sigma}(\boldsymbol{H}): \boldsymbol{\epsilon}(\overline{\boldsymbol{z}}) \mathrm{d} \boldsymbol{x}+\int_{\Omega_{f}^{\varepsilon}} \boldsymbol{f} \cdot \overline{\boldsymbol{z}} \mathrm{d} \boldsymbol{x},
$$

hence,

$$
\alpha\|\overline{\boldsymbol{z}}\|_{H^{1}\left(\Omega ; \mathbb{R}^{2}\right)}^{2} \leq\|\boldsymbol{g}\|_{H^{-\frac{1}{2}\left(\Gamma^{\varepsilon} ; \mathbb{R}^{2}\right)}}\|\overline{\boldsymbol{z}}\|_{H^{\frac{1}{2}\left(\Gamma^{\varepsilon} ; \mathbb{R}^{2}\right)}}+\|\boldsymbol{f}\|_{H^{-1}\left(\Omega ; \mathbb{R}^{2}\right)}\|\overline{\boldsymbol{z}}\|_{H^{1}\left(\Omega ; \mathbb{R}^{2}\right)},
$$

and therefore,

$$
\alpha\|\overline{\boldsymbol{z}}\|_{H^{1}\left(\Omega ; \mathbb{R}^{2}\right)}^{2} \leq C\left(\|\boldsymbol{g}\|_{H^{-\frac{1}{2}\left(\Gamma^{\varepsilon} ; \mathbb{R}^{2}\right)}}+\|\boldsymbol{H}\|_{H^{1}\left(\Omega ; \mathbb{R}^{2}\right)}+\|\boldsymbol{f}\|_{H^{-1}\left(\Omega ; \mathbb{R}^{2}\right)}\right)\|\overline{\boldsymbol{z}}\|_{H^{1}\left(\Omega ; \mathbb{R}^{2}\right)} .
$$

The continuity of the lifting operator gives us that $\|\boldsymbol{H}\|_{H^{1}\left(\Omega ; \mathbb{R}^{2}\right)} \leq C\|\boldsymbol{h}\|_{H^{\frac{1}{2}}\left(\Gamma ; \mathbb{R}^{2}\right)}$, so we can write:

$$
\alpha\|\overline{\boldsymbol{z}}\|_{H^{1}\left(\Omega ; \mathbb{R}^{2}\right)} \leq C\left(\|\boldsymbol{g}\|_{H^{-\frac{1}{2}\left(\Gamma^{\varepsilon} ; \mathbb{R}^{2}\right)}}+\|\boldsymbol{h}\|_{H^{\frac{1}{2}\left(\Gamma ; \mathbb{R}^{2}\right)}}+\|\boldsymbol{f}\|_{H^{-1}\left(\Omega ; \mathbb{R}^{2}\right)}\right) .
$$

We can now state the following result:

Theorem 4.2. In the isotropic case $\boldsymbol{E}_{m}: \boldsymbol{\epsilon}=\lambda$ trace $(\boldsymbol{\epsilon}) I+2 \mu \boldsymbol{\epsilon}$, there exists a constant $C>0$ independent of $\varepsilon$ such that:

$$
\left\|\boldsymbol{d}_{K}^{\varepsilon}\right\|_{H^{1}\left(\Omega ; \mathbb{R}^{2}\right)} \leq C \varepsilon^{K+1}, \quad \text { for all } K \in \mathbb{N} .
$$


Proof. With $\boldsymbol{z}=\boldsymbol{d}_{K}^{\varepsilon}$ we have $\boldsymbol{f} \in O_{H^{-1}\left(\Omega ; \mathbb{R}^{2}\right)}\left(\varepsilon^{K+1}\right), \boldsymbol{g} \in O_{H^{-\frac{1}{2}}\left(\Gamma^{\varepsilon}, \mathbb{R}^{2}\right)}\left(\varepsilon^{K+1}\right)$ and $\boldsymbol{h} \in O_{H^{\frac{1}{2}}\left(\Gamma, \mathbb{R}^{2}\right)}\left(\varepsilon^{K+1}\right)$ using Lemma 4.1 we obtain the announced error estimate.

Remark 4.3. The limitation of the error estimate to the isotropic case is only due to the limitation to this case of the expansion at infinity given in Section 4.3. An extension to some non-isotropic cases may require an adapted result of expansion at infinity of functions $\boldsymbol{V}_{m}^{(p)}$ and $\boldsymbol{Z}_{m}^{(p)}$.

\section{Conclusion and perspectives}

The purpose of this work was to study the interaction between an inclusion of a small size compared to the size of the elastic body in which it operates. We were able to build an asymptotic expansion starting from the field without inclusion and correcting by additional functions that model the presence of the inclusion and its influence on the mechanical fields (asymptotic expansion at an arbitrary order $K$ given by $u_{\varepsilon}^{[K]}(\boldsymbol{x})$ in (3.24) for the Poisson problem and by the displacement $\boldsymbol{u}_{\varepsilon}^{[K]}(\boldsymbol{x})$ in (4.27) for linear elasticity). The model had been validated by comparing the obtained result given by (3.51) with the one obtained by solving the problem using a separation of variable method (3.47) (for the Poisson problem and with a circular shape of the inclusion). Error estimates had been established in Sections 3.4 and 4.4. The main result for the applications is that the functions $v^{(i)}$ and $V^{(i)}$, solutions to some problems do not depend on $\varepsilon$ and depend linearly on $u^{(0)}$ only by the expansion of its gradient at $x=0$. For a given shape of the inclusion, this make it possible to pre-compute once for all a basis for each $v^{(i)}$ and $V^{(i)}$.

Natural perspectives of this work is the design of a numerical strategy first to compute a few terms of the expansion obtained and then to compute the influence of an inclusion in an arbitrary shaped body and the influence of several inclusions. Extension to large strain elasticity and to three-dimensional problems are also of great interest. Finally, the extension to inhomogeneous and anisotropic cases should be done without major difficulties.

\section{Acknowledgments}

This work was funded by a grant from the french manufacturer of tire "Michelin" and contributions from the Franco-Tunisian project PHC-Utique CMCU No. 14G1103, Campus France No. 30643PM.

Special thanks to Patrice Hauret, Thomas Homolle and Eric Lignon from the Michelin research and development team for fruitful exchanges and constructive advices during multiple meetings.

\section{Appendix}

\section{A Poincaré-type inequality}

We establish the existence and uniqueness of the profile functions $V^{(k)}$ introduced previously in the asymptotic expansion at order $K$ given by (3.24). The functions $V^{(k)}$ are defined on $\mathbb{R}^{2}$ (two-dimensional geometry) and are solutions to the family of Problems (3.5). For that, we will introduce weighted Sobolev spaces $\mathcal{W}_{\alpha, \beta}^{m, p}\left(\mathcal{W}_{0,0}^{1,2}\right.$ in our case) similar to classical ones $\mathcal{W}^{m, p}\left(H^{1}\right.$ in our case) but with weights that describe the growth or the decay of functions at infinity. 
The idea of using weighted spaces arises naturally from Hardy's inequalities and will allow us to establish a Poincaré inequality relating to norms of functions and to that of their derivatives.

The use of these weights is necessary to obtain a Poincaré-type inequality and eliminates the drawbacks of spaces defined by the closure of $\mathcal{D}\left(\mathbb{R}^{2}\right)$ for the Dirichlet norm and which are not always spaces of distributions. The used weights arises naturally from Hardy's inequality or from a generalized Hardy's inequality (see [31]) and the classical ones are of the form $\rho=\left(1+|x|^{2}\right)^{\frac{1}{2}}$ but there is appearance of a logarithmic factor in our case.

\section{A.1 Notations and functional setting}

All functions and distributions here are defined on the two-dimensional real Euclidean space $\mathbb{R}^{2}$.

Let $r=|x|=\left(x_{1}^{2}+x_{2}^{2}\right)^{\frac{1}{2}}$ be the distance of a point $x=\left(x_{1}, x_{2}\right)$ to the origin. Recall that $\mathcal{D}\left(\mathbb{R}^{2}\right)$ denotes the space of infinitely differentiable functions with compact support and $\mathcal{D}^{\prime}\left(\mathbb{R}^{2}\right)$ its dual space called space of distributions. With $\lambda=\left(\lambda_{1}, \ldots, \lambda_{n}\right) \in \mathbb{N}^{n}$ a multi-index $D^{\lambda}=D_{1}^{\lambda_{1}} \cdots D_{n}^{\lambda_{n}}$ is the differential operator of order $|\lambda|=\lambda_{1}+\cdots+\lambda_{n} . L^{2}\left(\mathbb{R}^{2}\right)$ is the classical space of measurable functions for which $\left(\int_{\mathbb{R}^{2}}|u|^{2} \mathrm{~d} \boldsymbol{x}\right)<\infty$. It is a Banach space for the norm $\|u\|=\left(\int_{\mathbb{R}^{2}}|u|^{2} \mathrm{~d} \boldsymbol{x}\right)^{\frac{1}{2}}$.

With $\rho=\left(1+r^{2}\right)^{\frac{1}{2}}$ we introduce the weighted Sobolev space, which is appropriate to our case, by:

$$
\mathcal{W}_{0,0}^{1,2}\left(\mathbb{R}^{2}\right)=\left\{u \in \mathcal{D}^{\prime}\left(\mathbb{R}^{2}\right), \rho^{-1}(\lg \rho)^{-1} u \in L^{2}\left(\mathbb{R}^{2}\right), \nabla u \in\left(L^{2}\left(\mathbb{R}^{2}\right)\right)^{2}\right\},
$$

which is a reflexive Banach space equipped with its natural norm:

$$
\|u\|_{\mathcal{W}_{0,0}^{1,2}\left(\mathbb{R}^{2}\right)}=\left(\left\|\rho^{-1}(\lg \rho)^{-1} u\right\|_{L^{2}\left(\mathbb{R}^{2}\right)}^{2}+\|\nabla u\|_{L^{2}\left(\mathbb{R}^{2}\right)}^{2}\right)^{\frac{1}{2}}
$$

We also define the semi-norm:

$$
|u|_{\mathcal{W}_{0,0}^{1,2}\left(\mathbb{R}^{2}\right)}=\|\nabla u\|_{L^{2}\left(\mathbb{R}^{2}\right)} .
$$

With $r=|x|$ we set $\lg (r)=\ln \left(2+r^{2}\right), B_{R}=B(0, R)$ the open ball with center 0 and radius $R$ and $B_{R}^{\prime}=\left(\bar{B}_{R}\right)^{c}$ the exterior of $\bar{B}_{R}$. Finally, we define $P_{0}$ as the space of constant functions.

\section{A.2 An intermediate result}

The following result is an intermediate result to prove the equivalence of the norm and the semi-norm.

Lemma A.1. For any large enough real number $R$, there exists a constant $C_{R}$ such that:

$$
\forall \varphi \in \mathcal{D}\left(B_{R}^{\prime}\right), \quad\|\varphi\|_{\mathcal{W}_{0,0}^{1,2}\left(B_{R}^{\prime}\right)} \leq C_{R}|\varphi|_{\mathcal{W}_{0,0}^{1,2}\left(B_{R}^{\prime}\right)} .
$$

Proof. Let $\varphi$ belongs to $\mathcal{D}_{R}^{\prime}$. First, observe that, owing to the support of $\varphi$, all integrals in the norm and semi-norm are taken on $B_{R}^{\prime}$ instead of $\mathbb{R}^{2}$. Hence, since the origin is in the interior of $B_{R}$, we can use $r$ and $\ln r$ instead of $\rho(r)$ and $\lg (r)$ in the expression of the norm and seminorm. Then using $\frac{\partial \varphi}{\partial r}=\nabla \varphi \cdot \frac{\mathbf{r}}{r}$ we can write:

$$
\left|\frac{\partial \varphi}{\partial r}\right|^{2} \leq 2 \sum_{i=1}^{2}\left|\frac{\partial \varphi}{\partial x_{i}}\right|^{2} .
$$

Let $\theta$ be the angular variable, then we have:

$$
\varphi(r, \theta)=\int_{R}^{r} \varphi(t, \theta) \mathrm{d} t .
$$


Now, assuming that $R$ is large enough, we apply the generalized Hardy's inequality (see [31]) (with $\gamma=-2$ ) to the function $r \rightarrow \varphi(r, \theta)$. Integrating with respect to $\theta$ and applying (A.1) we obtain:

$$
\left\|r^{-1} \ln r^{-1} \varphi\right\|_{L^{2}\left(B_{R}^{\prime}\right)} \leq C\|D \varphi\|_{L^{2}\left(B_{R}^{\prime}\right)} .
$$

The needed result can now be proven.

\section{A.3 A Poincaré-type inequality}

Theorem A.2. The semi-norm $|\cdot|_{\mathcal{W}_{0,0}^{1,2}\left(\mathbb{R}^{2}\right)}$ defines on $\mathcal{W}_{0,0}^{1,2}\left(\mathbb{R}^{2}\right) / P_{0}$ a norm which is equivalent to the quotient norm.

Proof. It is clear that $|\cdot|_{\mathcal{W}_{0,0}^{1,2}\left(\mathbb{R}^{2}\right)}$ is a norm on $\mathcal{W}_{0,0}^{1,2}\left(\mathbb{R}^{2}\right) / P_{0}$, and that:

$$
\forall u \in \mathcal{W}_{0,0}^{1,2}\left(\mathbb{R}^{2}\right), \quad|u|_{\mathcal{W}_{0,0}^{1,2}}\left(\mathbb{R}^{2}\right) \leq\|u\|_{\mathcal{W}_{0,0}^{1,2}}\left(\mathbb{R}^{2}\right)
$$

Thus, we only have to prove that there exits $c>0$ such that:

$$
\forall \dot{u} \in \mathcal{W}_{0,0}^{1,2}\left(\mathbb{R}^{2}\right) / P_{0}, \quad\|\dot{u}\|_{\mathcal{W}_{0,0}^{1,2}\left(\mathbb{R}^{2}\right) / P_{0}} \leq c|u|_{\mathcal{W}_{0,0}^{1,2}}\left(\mathbb{R}^{2}\right)
$$

The proof proceeds in two steps. The first step consists in eliminating the quotient norm by choosing an adequate representative of the class of $\dot{u}$. To this end, we fix a bounded open domain of $\mathbb{R}^{2}$, with positive measure, say $O$, and we choose the representative $U$ of $\dot{u}$ in $\mathcal{W}_{0,0}^{1,2}\left(\mathbb{R}^{2}\right)$ that satisfies the system of equations:

$$
\forall \mu \in P_{0}, \int_{O} U \mu \mathrm{d} \boldsymbol{x}=0 .
$$

It is easy to see that (A.4) determines $U$ uniquely and that:

$$
\|\dot{u}\|_{\mathcal{W}_{0,0}^{1,2}\left(\mathbb{R}^{2}\right) / P_{0}} \leq\|U\|_{\mathcal{W}_{0,0}^{1,2}\left(\mathbb{R}^{2}\right)} .
$$

Therefore, the second step consists in proving that there exists a constant $C$ such that the following bound holds for all $U$ in $\mathcal{W}_{0,0}^{1,2}\left(\mathbb{R}^{2}\right)$ satisfying (A.4):

$$
\|U\|_{\mathcal{W}_{0,0}^{1,2}\left(\mathbb{R}^{2}\right)} \leq C|U|_{\mathcal{W}_{0,0}^{1,2}\left(\mathbb{R}^{2}\right)} .
$$

We shall prove it by contradiction. If (A.6) is not true, there exists a sequence $\left(U_{\nu}\right)$ of elements of $\mathcal{W}_{0,0}^{1,2}\left(\mathbb{R}^{2}\right)$ satisfying (A.5) and such that:

$$
\left\|U_{\nu}\right\|_{\mathcal{W}_{0,0}^{1,2}\left(\mathbb{R}^{2}\right)}=1 \text { and }\left|U_{\nu}\right|_{\mathcal{W}_{0,0}^{1,2}\left(\mathbb{R}^{2}\right)} \rightarrow 0
$$

Hence the sequence $\left(U_{\nu}\right)$ is bounded in $\mathcal{W}_{0,0}^{1,2}\left(\mathbb{R}^{2}\right)$ and since this is a reflexive Banach space, we can extract a subsequence, still denoted by $\left(U_{\nu}\right)$, that converges weakly to an element $U_{*}$ of $\mathcal{W}_{0,0}^{1,2}\left(\mathbb{R}^{2}\right)$ and it is easy to check from this weak convergence that $U_{*}$ also satisfies (A.5). But since $|u|_{\mathcal{W}_{0,0}^{1,2}\left(\mathbb{R}^{2}\right)}$ tends to 0 , the lower semi-continuity of the norm implies that $|u|_{\mathcal{W}_{0,0}^{1,2}\left(\mathbb{R}^{2}\right)}=0$. Thus, $U_{*}$ is a constant (polynomial of $P_{0}$ ) and the fact that $U_{*}$ satisfies (A.5) implies that $U_{*}=0$.

Now, we need a strong convergence to conclude by contradiction, but we cannot use a standard compactness argument on an unbounded domain. Instead, we shall derive a strong convergence 
via an adequate partition of unity that will enable us to consider separately a bounded domain where the topologies of $\mathcal{W}_{0,0}^{1,2}$ and $H^{1}$ coincide and the exterior of a ball, where Lemma A.1 can be applied.

Let $R$ denote a real number, large enough to apply the generalized Hardy's inequality. Let $\varphi$ and $\psi$ be two functions of $C^{\infty}\left(\mathbb{R}^{2}\right)$ such that:

$$
\forall \boldsymbol{x} \in \mathbb{R}^{2},(\varphi+\psi)(\boldsymbol{x})=1,0 \leq \varphi(\boldsymbol{x}) \leq 1, \operatorname{supp}(\varphi) \subset \bar{B}_{R+1}, \operatorname{supp}(\psi) \subset B_{R}^{\prime} .
$$

Since for fixed $R, \mathcal{W}_{0,0}^{1,2}\left(B_{R+1}\right)$ is isomorphic to $R, H^{1}\left(B_{R+1}\right)$, we have that $U_{\mu}$ converges weakly to 0 in $H^{1}\left(B_{R+1}\right)$. Since $H^{1}\left(B_{R+1}\right)$ is compactly embedded into $L^{2}\left(B_{R+1}\right)$, it follows that

$$
U_{\nu} \rightarrow 0 \text { strongly in } L^{2}\left(B_{R+1}\right) \text {, }
$$

In addition, as $\left|U_{\nu}\right|_{\mathcal{W}_{0,0}^{1,2}\left(\mathbb{R}^{2}\right)}$ tends to 0 , it follows that

$$
U_{\nu} \rightarrow 0 \text { strongly in } \mathcal{W}^{1,2}\left(B_{R+1}\right)
$$

so that

$$
\varphi U_{\nu} \rightarrow 0 \text { strongly in } \mathcal{W}_{0,0}^{1,2}\left(B_{R+1}\right)
$$

Now, let us examine the behavior of $\psi U_{\nu}$. For fixed $\nu$, let $\left(\theta_{j}\right)$ be a sequence of functions of $\mathcal{D}\left(\mathbb{R}^{2}\right)$ that tends to $U_{\nu}$ in $\mathcal{W}_{0,0}^{1,2}\left(\mathbb{R}^{2}\right)$. Then, $\psi \theta_{j}$ belongs to $\mathcal{D}\left(B_{R}^{\prime}\right)$ and we can apply to it Lemma (A.1)

$$
\left\|\psi \theta_{j}\right\|_{\mathcal{W}_{0,0}^{1,2}\left(\mathbb{R}^{2}\right)} \leq C_{R} \mid \psi \theta_{j} \|_{\mathcal{W}_{0,0}^{1,2}\left(B_{R}^{\prime}\right)}{ }
$$

Then, letting $j$ tend to infinity and using the fact that $\psi$ is identically one outside $B_{R+1}$, we obtain:

$$
\left\|\psi U_{\nu}\right\|_{\mathcal{W}_{0,0}^{1,2}\left(\mathbb{R}^{2}\right)} \leq C_{R}\left|\psi U_{\nu}\right|_{\mathcal{W}_{0,0}^{1,2}\left(B_{R}^{\prime}\right)} \leq C_{R}\left(\left|\psi U_{\nu}\right|_{\mathcal{W}_{0,0}^{1,2}\left(B_{R}^{\prime} \cap B_{R+1}\right)}^{p}+\left|U_{\nu}\right|_{\mathcal{W}_{0,0}^{1,2}\left(B_{R+1}^{\prime}\right)}^{p}\right)^{\frac{1}{p}}
$$

Next, let $\nu$ tend to infinity and observe that $\psi U_{\nu}$ tends to zero strongly in $\mathcal{W}_{0,0}^{1,2}\left(B_{R}^{\prime} \cap B_{R+1}\right)$ because $B_{R}^{\prime} \cap B_{R+1}$ is bounded and $\mathcal{W}_{0,0}^{1,2}\left(B_{R}^{\prime} \cap B_{R+1}\right)$ is isomorphic to $\mathcal{W}^{1,2}\left(B_{R}^{\prime} \cap B_{R+1}\right)$; we derive that:

$$
\psi U_{\nu} \rightarrow 0 \text { strongly in } \mathcal{W}_{0,0}^{1,2}\left(B_{R}^{\prime}\right)
$$

Since $U_{\nu}=\varphi U_{\mu}+\psi U_{\mu}$, we obtain:

$$
U_{\mu} \rightarrow 0 \text { strongly in } \mathcal{W}_{0,0}^{1,2}\left(\mathbb{R}^{2}\right),
$$

which contradicts the assumption (A.7) that:

$$
\left\|U_{\mu}\right\|_{\mathcal{W}_{0,0}^{1,2}\left(\mathbb{R}^{2}\right)}=1 .
$$




\section{References}

[1] E. Lignon, Modélisation multi-échelles de nappes fibrées en compression, Ph.D. thesis, Ecole Polytechnique (2011).

[2] G. Vial, Analyse multi-échelle et conditions aux limites approchées pour un probleme avec couche mince dans un domaine à coin, Ph.D. thesis, Université de Rennes 1 (2003).

[3] M. Dambrine and G. Vial, Influence of a boundary perforation on a Dirichlet energy, Control and Cybernetics, 34(1), (2005), 117-136.

[4] E. Sánchez-Palencia, Problèmes de perturbations liés aux phénomènes de conduction à travers des couches minces de grande résistivité, J. Math. Pures Appl., 53(9), (1974), 251269.

[5] A. Bendali and K. Lemrabet, The effect of a thin coating on the scattering of a time-harmonic wave for the Helmholtz equation, SIAM J. Appl. Math., 56(6), (1996), 1664-1693.

[6] D. J. Hansen, C. Poignard, and M. S. Vogelius, Asymptotically precise norm estimates of scattering from a small circular inhomogeneity, Appl. Anal., 86(4), (2007), 433-458.

[7] G. Geymonat, F. Krasucki, and S. Lenci, Mathematical analysis of a bonded joint with a soft thin adhesive, Math. Mech. Solids, 4(2), (1999), 201-225.

[8] V. Maz'ya, S. Nazarov, and B. Plamenevskij, Asymptotic Theory of Elliptic Boundary Value Problems in Singularly Perturbed Domains, Birkhauser Verlag, Basel (2000).

[9] - Asymptotic Theory of Elliptic Boundary Value Problems in Singularly Perturbed Domains., Birkhauser Verlag, Basel (2000).

[10] A. M. Il'in, Matching of Asymptotic Expansions of Solutions of Boundary Value Problems, Amer. Math. Soc., Providence RI (1992).

[11] T. Lewinski and J. Sokolowski, Topological derivative for nucleation of non-circular voids. The Neumann problem, in R. Gulliver, W. Littman, and R. Triggiani, editors, Differential Geometric Methods in the Control of Partial Differential Equations, volume 268, Amer. Math. Soc., Providence, RI (2000), 341-361.

[12] M. Dambrine and G. Vial, A multiscale correction method for local singular perturbations of the boundary, Math. Model. Numer. Anal., 41(1), (2007), 111-127.

[13] J. D. Eshelby, The determination of the elastic field of an ellipsoidal inclusion, and related problems, Proceedings of the Royal Society of London. Series A, Mathematical and Physical Sciences, 241, (1957), 376-396.

[14] - , The elastic field outside an ellipsoidal inclusion, Proceedings of the Royal Society of London. Series A, Mathematical and Physical Sciences, 252, (1959), 561-569.

[15] P. Schiavone, Neutrality of the elliptic inhomogeneity in the case of non-uniform loading, IMA journal of applied mathematics, 8, (2003), 161-169.

[16] Y. Antipov and P. Schiavone, On the uniformity of stresses inside an inhomogeneity of arbitrary shape, Int. J. Eng. Sci., 68, (2003), 299-311. 
[17] S. Li, R. Sauer, and G. Wang, A circular inclusion in a finite domain I. The DirichletEshelby problem, Acta Mechanica, 179(1), (2005), 67-90.

[18] E. Kröner, Statistical Modelling, in J. Gittus and J. Zarka, editors, Modelling Small Deformations of Polycrystals, Springer, Dordrecht, the Netherlands (1986), 229-291.

[19] -, Modified Green Functions in the Theory of Heterogeneous and/or Anisotropic Linearly Elastic Media, in G. J. Weng, M. Taya, and H. Abé, editors, Micromechanics and Inhomogeneity: The Toshio Mura 65th Anniversary Volume, Springer, New York, NY (1990), $197-211$.

[20] P. Mazilu, On the theory of linear elasticity in statically homogeneous media, Rev. Roum. Math. Pur. Appl., 17, (1972), 261-273.

[21] H. Ammari and H. Kang, Reconstruction of small inhomogeneities from boundary measurements, Springer-Verlag, Berlin Heidelberg (2004).

[22] C. S. Chand and H. D. Conway, A parametric study of the complex variable method for analyzing the stresses in an infinite plate containing a rigid rectangular inclusion, Int. J. Solids Structures, 4, (1968), 1057-1066.

[23] Y. Sun and Y. Peng, Analytic solutions for the problems of an inclusion of arbitrary shape embedded in a half space, Applied Mathematics and Computation, 140, (2003), 105-113.

[24] E. Beretta, E. Bonnetier, E. Francini, and A. L. Mazzucato, An asymptotic formula for the displacement field in the presence of small anisotropic elastic inclusions, Inverse Problems and Imaging, 6, (2012), 1-23.

[25] Y. Capdeboscq and M. Vogelius, A general representation formula for boundary voltage perturbations caused by internal conductivity inhomogeneities of low volume fraction, Math. Modelling Num. Anal., 37, (2003), 159-173.

[26] - , A review of some recent work on impedance imaging for inhomogeneities of low volume fraction, Contemp. Math., 362, (2004), 69-87.

[27] E. Bonnetier and F. Triki, Asymptotics in the presence of inclusions of small volume for a conduction equation: A case with a non-smooth reference potential, Contemporary Mathematics, 494, (2009), 95-107.

[28] A. Ern and J.-L. Guermond, Theory and Practice of Finite Elements, Springer-Verlag, New York (2004).

[29] A. Fortin and A. Garon, Les Eléments Finis : de la Théorie à la Pratique, Université Laval Ecole Polytechnique de Montréal, Montreal (2011).

[30] H. Brezis, Progress in Nonlinear Differential Equations and Their Applications: In memory of Pierre Grisvard, Birkhäuser, Boston (1996).

[31] C. Amrouche, V. Girault, and J. Giroire, Weighted Sobolev spaces for Laplace's equation in $\mathbb{R}^{n}$, J. Math. Pures Appl., 73, (1994), 579-606. 